\title{
Emergent biaxiality in nematic microflows illuminated by a laser beam
}

\author{
Jan-Christoph Eichler, Robert A. Skutnik, Anupam Sengupta, Marco G. Mazza \\ \& Martin Schoen
}

To cite this article: Jan-Christoph Eichler, Robert A. Skutnik, Anupam Sengupta, Marco G. Mazza \& Martin Schoen (2019) Emergent biaxiality in nematic microflows illuminated by a laser beam, Molecular Physics, 117:23-24, 3715-3733, DOI: 10.1080/00268976.2019.1663286

To link to this article: https://doi.org/10.1080/00268976.2019.1663286

View supplementary material $\widetilde{ }$

曲 Published online: 16 Sep 2019.

एँ⿱

Submit your article to this journal

Џll Article views: 81

Q View related articles $\square$

View Crossmark data $\asymp$ 


\title{
Emergent biaxiality in nematic microflows illuminated by a laser beam
}

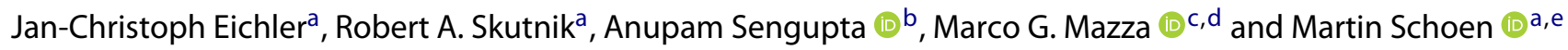

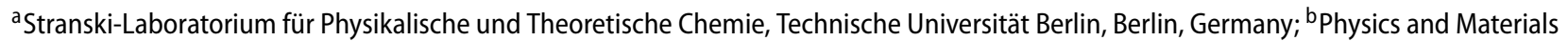 \\ Science Research Unit, University of Luxembourg, Luxembourg City, Grand Duchy of Luxembourg; ${ }^{C}$ Interdisciplinary Centre for Mathematical \\ Modelling and Department of Mathematical Sciences, Loughborough University, Loughborough, UK; ${ }^{d}$ Max Planck Institute for Dynamics and \\ Self-Organization (MPIDS), Göttingen, Germany; ${ }^{\text {}}$ Department of Chemical Engineering, Imperial College London, London, UK
}

\begin{abstract}
Anisotropic fluids (e.g. liquid crystals) offer a remarkable promise as optofluidic materials owing to the directional, tunable, and coupled interactions between the material, flow, and the optical fields. Here we present a comprehensive in silico treatment of this anisotropic interaction by performing nonequilibrium molecular dynamics simulations. We quantify the response of a nematic liquid crystal (NLC) undergoing a Poiseuille flow in the Stokes regime, while being illuminated by a laser beam incident perpendicular to the flow direction. We adopt a minimalistic model to capture the interactions, accounting for two features: first, the laser heats up the NLC locally; and second, the laser polarises the NLC and exerts an optical torque that tends to reorient molecules of the nematic phase. Because of this reorientation the liquid crystal exhibits small regions of biaxiality, where the nematic director is one symmetry axis and the axis of rotation for the reorientation of the molecules is the other one. We find that the relative strength of the viscous and the optical torques mediates the flow-induced response of the biaxial regions, thereby tuning the emergence, shape and location of the regions of enhanced biaxiality. The mechanistic framework presented here promises experimentally tractable routes toward novel optofluidic applications based on material-flow-light interactions.
\end{abstract}

ARTICLE HISTORY

Received 5 June 2019

Accepted 27 August 2019

\section{KEYWORDS}

Liquid crystal; nematic phase; optofluidics; biaxiality; nonequilibrium molecular dynamics

\section{Introduction}

Liquid crystals represent a nearly unique arena where thermodynamics, hydrodynamics, and symmetry come together to produce remarkable effects, completely absent in matter composed of molecules with isotropic interactions. Liquid crystals flow, and exhibit molecular order; they mediate interactions among colloids, and have essential electro-optical properties.

Nematic liquid crystals are typically considered the simplest member of their class. Composed of elongated molecules, molecular interactions lead to a preferential parallel alignment that produces a spontaneous breaking of the continuous rotational symmetry. A thermodynamic phase with a preferential axis, called nematic director, emerges. The long range orientational order in nematic liquid crystals leads to intrinsic anisotropy in the material properties [1], resulting in directional response to applied external fields (e.g. electric, magnetic, optical or viscous). The higher the anisotropy, the stronger is the strength of the coupling between the nematic director and the external field. 
Such anisotropic couplings have been traditionally utilised to develop a range of liquid crystal based applications [2], and more recently, lab-on-a-chip concepts that harness the dynamics of liquid crystal flows under microfluidic settings [3-7]. Despite the rapid progress in liquid crystal microfluidics, the integration of optical field to such platforms is still in its infancy. Optical fields have been extensively applied to modulate properties of isotropic fluids flowing within microscale confinements. This is evidenced by the emergence of optofluidics, an active and well-established field of interdisciplinary research [8]. However, replacing an isotropic fluid by an anisotropic flowing material (e.g. liquid crystals) could offer promising alternatives, and usher in unique applications based on the coupled, tunable and anisotropic attributes [9-11]. Yet, to date, we lack a physical understanding of how - in conjunction - the flow, director and optical fields interact and emerge. The flow-directoroptical nexus is a hallmark of liquid crystal based optofluidics, and thus warrants a comprehensive theoretical treatment that could enable innovative applications in future.

For nematics, the statistical distribution of the molecular orientations is independent of the angle about their long axes; the system has uniaxial symmetry. Consider now a liquid whose molecular building blocks are shaped as parallelepipeds. The distribution of the molecular orientations will show a strong correlation in a direction perpendicular to the nematic director. This so-called biaxial fluid thus exhibits two symmetry axes: the director and the secondary axis. First theoretically predicted in 1970 by Freiser [12], biaxial nematic phases have spurred a controversial experimental hunt [13-17] and numerous theoretical studies [18-25], because such liquids would have fast response times under varying electric fields, and thus would be of particular relevance for display technologies. Concrete realisations of biaxial nematics have mostly explored two main avenues: (i) sophisticated chemical tuning of molecular shape or interactions among basic moieties [26,27]; (ii) variation of the solvent concentration in lyotropic liquid crystals [28].

Liquid crystals, however, offer the remarkable liberty of manipulating the topology of their alignment field by means of the hydrodynamic coupling of the director field and advective velocity [29-31]. One of the main physical processes of liquid crystals (captured by the Ericksen-Leslie theory or more sophisticated approaches such as the Beris-Edwards or the Qian-Sheng nematodynamics) is that a local flow velocity will induce a change of orientations, and, conversely, a local change of orientations will induce local flows.

Application of an external electric field provides a direct way of controlling the nematic director, on account of the dipolar moment of liquid crystal molecules. The ability of light to control the nematic director is the basis of the modulation of the index of refraction that makes liquid crystals so successful in display technologies. However, electric fields may also produce complex nonlinear behaviour in liquid crystals.

We can then envision that the combined effect of anchoring condition to a solid wall, flow, and possibly an external field may induce the formation of a secondary axis in a nematic fluid that in equilibrium conditions would be uniaxial, and thus effectively producing biaxiality in steady-state nonequilibrium conditions. In this work we begin to explore the setup of a nematic phase in a model microfluidic setup. We confine a uniaxial nematic fluid between two solid walls. We establish a steady-state flow by driving the fluid in the microfluidic channel. Furthermore, we expose a section of the system to a model laser that has both a thermal effect and also a realigning torque.

The remainder of this work is organised as follows. Section 2 describes our models for the liquid crystal, external fields, and confining walls. Section 3 introduces the main observables that we will use to characterise our system. Section 4 gives details of the numerical scheme used to simulate our nematic microfluidic setup. Section 5 reports our results. In Section 6 we discuss our results and collect our conclusions. Finally, the Appendices show details of the anisotropic interaction potential and of the local alignment tensor.

\section{Model}

\subsection{Potentials}

Consider a liquid crystal composed of $N$ uniaxial molecules (i.e. mesogens) interacting with each other in a pairwise additive fashion. We formally split the interaction potential into an isotropic and into an anisotropic contribution according to

$$
u\left(\boldsymbol{r}_{i j}, \omega_{i}, \omega_{j}\right)=u_{\text {iso }}\left(r_{i j}\right)+u_{\text {aniso }}\left(\boldsymbol{r}_{i j}, \omega_{i}, \omega_{j}\right),
$$

where $\boldsymbol{r}_{i j}=\boldsymbol{r}_{i}-\boldsymbol{r}_{j}$ is the distance vector connecting the centres of mass of mesogens $i$ and $j$. They are located at the respective positions $\boldsymbol{r}_{i}$ and $\boldsymbol{r}_{j}$ and $r_{i j}=\left|\boldsymbol{r}_{i j}\right|$. Because we assume the mesogens to be uniaxial, their orientation can be specified by Euler angles $\omega_{i}=\left(\theta_{i}, \varphi_{i}\right) \quad(i=$ $1, \ldots, N)$ where $\theta_{i}$ and $\varphi_{i}$ denote polar and azimuthal angle, respectively.

For later reference we emphasise that both angles refer to a Cartesian coordinate system characterised by the mutually orthonormal unit vectors, $\widehat{\boldsymbol{e}}_{\mathrm{x}}^{\mathrm{T}}=(1,0,0), \widehat{\boldsymbol{e}}_{\mathrm{y}}^{\mathrm{T}}=$ $(0,1,0)$, and $\widehat{\boldsymbol{e}}_{\mathrm{z}}^{\mathrm{T}}=(0,0,1)$ where $\mathrm{T}$ denotes the transpose. We refer to the set $\left\{\widehat{\boldsymbol{e}}_{\mathrm{x}}, \widehat{\boldsymbol{e}}_{\mathrm{y}}, \widehat{\boldsymbol{e}}_{\mathrm{z}}\right\}$ as the standard basis. 
Throughout this manuscript the caret is used to indicate a unit vector. Hence, we can characterise the orientation of mesogen $i$ by

$$
\widehat{\boldsymbol{u}}_{i} \equiv \widehat{\boldsymbol{u}}\left(\omega_{i}\right)=\left(\begin{array}{c}
\sin \theta_{i} \cos \varphi_{i} \\
\sin \theta_{i} \sin \varphi_{i} \\
\cos \theta_{i}
\end{array}\right), \quad i=1, \ldots, N
$$

For the interaction between the spherically symmetric cores of the mesogens we adopt the purely repulsive softsphere potential

$$
u_{\text {iso }}\left(r_{i j}\right)=4 \varepsilon\left(\frac{\sigma}{r_{i j}}\right)^{12},
$$

where $\sigma$ is a characteristic length (see below) and $\varepsilon$ sets the energy scale for the isotropic repulsion between these cores.

To derive a tractable expression for the anisotropic contribution to the interaction potential we begin by noticing that any function depending on $\boldsymbol{r}_{i j}, \omega_{i}$, and $\omega_{j}$ can be expanded [see Sec. A.4.2 of Ref. [32]] according to

$$
\begin{aligned}
u_{\text {aniso }}\left(r_{i j}, \omega_{i}, \omega_{j}\right)= & \sum_{l_{1} l_{2} l} u_{l_{1} l_{2} l}\left(r_{i j}\right) \\
& \times \Phi_{l_{1} l_{2} l}\left(\omega_{i}, \omega_{j}, \omega_{i j}\right),
\end{aligned}
$$

where $\left\{u_{l_{1} l_{2} l}\right\}$ is a set of expansion coefficients that depend only on the centre-of-mass distance between a pair of mesogens and $\omega_{i j}$ specifies the orientation of $\widehat{\boldsymbol{r}}_{i j}=\boldsymbol{r}_{i j} / r_{i j}$ in a space-fixed frame of reference; $\tilde{l}$ (that is, $l_{1}, l_{2}$, or $l$ ) is a non-negative integer. In Equation (4)

$$
\begin{aligned}
\Phi_{l_{1} l_{2} l}\left(\omega_{i}, \omega_{j}, \omega_{i j}\right)= & \sum_{m_{1} m_{2} m} C\left(l_{1} l_{2} l ; m_{1} m_{2} m\right) \\
& \times \mathcal{Y}_{l_{1} m_{1}}\left(\omega_{i}\right) \mathcal{Y}_{l_{2} m_{2}}\left(\omega_{j}\right) \mathcal{Y}_{l m}^{*}\left(\omega_{i j}\right)
\end{aligned}
$$

is a rotational invariant where $C$ is a Clebsch-Gordan coefficient [32]; $\widetilde{m}$ (that is, $m_{1}, m_{2}$, or $m$ ) is an integer linked to the corresponding $\widetilde{l}$ through $\tilde{m} \in[-\widetilde{l}, \widetilde{l}]$. Thus, for each value of $\widetilde{l}$ there are $\tilde{2} \tilde{l}+1$ integers $\widetilde{m}$. In Equation (5), $\mathcal{Y}_{I_{\tilde{m}}}$ denotes a spherical harmonic and * indicates its complex conjugate.

Based upon this approach we eventually arrive at (see Appendix 1)

$$
\begin{aligned}
u\left(r_{i j}, \omega_{i}, \omega_{j}\right)= & 4 \varepsilon\left[\left(\frac{\sigma}{r_{i j}}\right)^{12}-\left(\frac{\sigma}{r_{i j}}\right)^{6}\right. \\
& \left.\times\left\{1+\Psi\left(\widehat{r}_{i j}, \omega_{i}, \omega_{j}\right)\right\}\right] \\
= & u_{\mathrm{LJ}}\left(r_{i j}\right)-\left(\frac{\sigma}{r_{i j}}\right)^{6} \Psi\left(\widehat{r}_{i j}, \omega_{i}, \omega_{j}\right),
\end{aligned}
$$

where the anisotropy function is given by the expression

$$
\begin{aligned}
\Psi\left(\widehat{\boldsymbol{r}}_{i j}, \omega_{i}, \omega_{j}\right)= & \varepsilon^{\prime} P_{2}\left(\widehat{\boldsymbol{u}}_{i} \cdot \widehat{\boldsymbol{u}}_{j}\right) \\
& +\varepsilon^{\prime \prime}\left[P_{2}\left(\widehat{\boldsymbol{u}}_{i} \cdot \widehat{\boldsymbol{r}}_{i j}\right)+P_{2}\left(\widehat{\boldsymbol{u}}_{j} \cdot \widehat{\boldsymbol{r}}_{i j}\right)\right]
\end{aligned}
$$

and $P_{2}(x)=\frac{1}{2}\left(3 x^{2}-1\right)$ is the second Legendre polynomial. Thus, we can define $\sigma$ in Equation (3) through the relation $u_{\mathrm{LJ}}(\sigma)=0$. In Equation (7), $\varepsilon^{\prime}$ and $\varepsilon^{\prime \prime}$ are dimensionless parameters rationalised in Appendix 1. In Appendix 1, we also describe how the expression for $\Psi$ in Equation (7) is derived.

It should perhaps be pointed out that the potential $u$ in Equation (6) with an anisotropy function $\Psi$ similar to the one given in Equation (7) has been presented earlier by Hess and $\mathrm{Su}$ [33]. Their model potential is derived through an expansion in terms of Wigner rotation matrices [34]. This approach is different from but equivalent to the one utilised in the present work.

For a suitable choice of values for the parameters $\varepsilon^{\prime}$ and $\varepsilon^{\prime \prime}$, Greschek et al. demonstrated that the potential defined by Equations (6) and (7) describes slightly elongated mesogens where a side-by-side arrangement of the longer axes of a pair is energetically preferred (see Fig. 1 of Ref. [35]). Throughout this work we employ $\varepsilon^{\prime}=0.21$ and $\varepsilon^{\prime \prime}=0.40$. These elongated mesogens can be described as ellipsoids of revolution characterised by a relatively small aspect ratio of 1.26 .

\subsection{Thermostat and external fields}

To study the model of a liquid crystal introduced in Section 2.1 under stationary nonequilibrium conditions we expose the $N$ mesogens to an external body force $\boldsymbol{F}_{\mathrm{e}}=F_{\mathrm{e}} \widehat{\boldsymbol{e}}_{\mathrm{x}}$ where $F_{\mathrm{e}}=\left|\boldsymbol{F}_{\mathrm{e}}\right|$. The body force is constantly applied in a small volume at one end of the volume $V$ occupied by the mesogens (see Section 4). Eventually, a steady laminar flow is established in the direction of $\boldsymbol{F}_{\mathrm{e}}$. To stay in the laminar flow regime, $F_{\mathrm{e}}$ obviously must not be too large.

On account of the application of $\boldsymbol{F}_{\mathrm{e}}$, which is nonconservative in nature, the liquid crystal would gradually heat up over time and thus would not reach a stationary nonequilibrium state. Therefore, we need to remove this excess heat due to $\boldsymbol{F}_{\mathrm{e}}$ from our system.

In non-equilibrium molecular dynamics (NEMD) simulations this can be accomplished by applying a thermostat. However, in a highly inhomogeneous system this can be a bit tricky because some global thermostats tend to remove too much heat from some regions of the inhomogeneous fluid whereas too little heat is removed from other parts [36]. 
As discussed in Ref. [36], a suitable thermostat to avoid these problems is the Galilean-invariant thermostat suggested by Stoyanov and Groot $[37,38]$. In this thermostat one considers an extra pair force

$$
\boldsymbol{F}_{i j}=\frac{\lambda \psi\left(r_{i j} / r_{\mathrm{c}}\right)}{\delta t}\left[1-\frac{1}{2} \frac{T_{i}-T_{j}}{T_{0}^{\prime}}\right]\left[\left(\boldsymbol{v}_{i}-\boldsymbol{v}_{j}\right) \cdot \widehat{\boldsymbol{r}}_{i j}\right] \widehat{\boldsymbol{r}}_{i j},
$$

where $\lambda=0.1 \pi$ denotes the strength of coupling to the thermostat, $\psi$ is a 'smearing' function (see below), $\delta t$ is the time step with which the centre-of-mass equations of motion are solved numerically, $T_{0}^{\prime}$ is the target temperature of the stationary nonequilibrium state, $\boldsymbol{v}_{i}$ and $\boldsymbol{v}_{j}$ are the translational velocities with which mesogens $i$ and $j$ move.

The quantities $T_{i}$ and $T_{j}$ in Equation (8) are somewhat peculiar temperatures of mesogens $i$ and $j$, respectively. They are affiliated with the difference in kinetic energies of the moving pair and are defined through the expression

$$
k_{\mathrm{B}} T_{i}=\frac{\sum_{j} \psi\left(r_{i j} / r_{\mathrm{c}}\right) m\left(\boldsymbol{v}_{i}-\boldsymbol{v}_{j}\right)^{2}}{3 \sum_{j} \psi\left(r_{i j} / r_{\mathrm{c}}\right)},
$$

where $m$ is the mass of a mesogen.

In Equation (8), the smearing function is somewhat arbitrary [37]. Here, we simply follow earlier work [37,39] and choose

$$
\psi(r)= \begin{cases}3(1-r) / \pi, & 0 \leq r \leq 1, \\ 0, & r \geq 1,\end{cases}
$$

where $r=r_{i j} / r_{\mathrm{c}}^{\prime}\left(r_{\mathrm{c}}^{\prime}=1.6 \sigma\right)$ and the factor $3 / \pi$ has been introduced to make sure that $4 \pi \int_{0}^{1} \psi(r) r^{2} \mathrm{~d} r=1$ [37]. The smearing function just serves as a means to ensure that the thermostat acts rather locally and not on larger length scales. For example, without the smearing function we would be back to a global thermostat which in a highly inhomogeneous system would most likely be prone to cause the aforementioned problems.

Once a steady flow is established through the protocol just described, a small portion of the liquid crystal is exposed to a temperature gradient and to an optical torque. Both perturbations are the most basic features of a laser illuminating that portion of the liquid crystal [40]. Our model laser causes the liquid crystal to heat up locally. We model this local heating by replacing the (constant) target temperature $T_{0}^{\prime}$ in Equation (8) by a local one via the equation

$$
T_{0}(x)=T_{\text {out }}+\left(T_{\text {in }}-T_{\text {out }}\right) \exp \left[-\frac{\alpha_{\mathrm{p}}^{2}}{2}\left(x-x_{0}\right)^{2}\right],
$$

which induces a temperature gradient in the flow (i.e. $x$-) direction. In Equation (11), $T_{\text {in }}$ and $T_{\text {out }}$ are the temperatures inside and outside of the illuminated region, respectively, $x_{0}$ is the position of the centre of the laser beam, and the inverse length $\alpha_{\mathrm{p}}$ is a measure of its width. Throughout this work we take $\alpha_{\mathrm{p}}=0.316 \sigma^{-1}\left(\alpha_{\mathrm{p}}^{2}=\right.$ $\left.0.1 \sigma^{-2}\right)$.

A second consequence of this illumination is that it exerts an optical torque on the uniaxial mesogens. Each mesogen $i$ therefore experiences a total torque that may be cast as

$$
\boldsymbol{\tau}_{i}=\boldsymbol{\tau}_{i}^{\mathrm{int}}+\boldsymbol{\tau}_{\text {opt }},
$$

where $\boldsymbol{\tau}_{i}^{\text {int }}$ is the total torque exerted on mesogen $i$ by other mesogens and

$$
\boldsymbol{\tau}_{\mathrm{opt}}=\varepsilon_{\mathrm{opt}} \exp \left[-\frac{\alpha_{\mathrm{p}}^{2}}{2}\left(x-x_{0}\right)^{2}\right] \widehat{\boldsymbol{p}} .
$$

In Equation (13), $\varepsilon_{\text {opt }}$ determines the coupling strength of the optical torque and $\widehat{\boldsymbol{p}}$ is given by

$$
\widehat{\boldsymbol{p}}=\left(\begin{array}{l}
0 \\
1 \\
0
\end{array}\right),
$$

which can be thought of as the direction in which the liquid crystal is polarised by the incoming laser beam. In Equations (11) and (13), $\alpha_{\mathrm{p}}$ is, of course, taken to be the same.

\subsection{Solid substrates}

In addition to the external field described in the preceding section we confine the liquid crystal by two planar solid substrates, each composed of a single plane of spherically symmetric particles; the walls are therefore atomically corrugated. The wall atoms are arranged in the $x-y$ plane according to the face-centred cubic (100) structure. The purpose of the substrates is to provide friction to the mesogens in their vicinity so that under steady-state conditions Poiseuille flow is established. The substrates are separated by a distance $s_{\mathrm{Z}}$ along the $z$-axis such that the lower one is placed at $z=-s_{z} / 2(k=1)$ whereas the upper one is located at $z=+s_{z} / 2(k=2)$.

The interaction between a mesogen and a substrate atom is described by the Lennard-Jones potential [cf., Equation (6)] that is

$$
u_{\mathrm{fs}}\left(r_{i j}^{(k)}\right)=4 \varepsilon_{\mathrm{fs}}\left[\left(\frac{\sigma}{r_{i j}^{(k)}}\right)^{12}-\left(\frac{\sigma}{r_{i j}^{(k)}}\right)^{6}\right],
$$

where $r_{i j}^{(k)}$ denotes the distance between the centres-ofmass of mesogen $i$ and a solid atom $j$ located in wall $k$. In 
this expression we tacitly assume that the spherical cores of both the mesogens and the solid atoms are of the same size proportional to $\sigma$. The depth of the attractive well is $\varepsilon_{\mathrm{fs}} / \varepsilon=2.0$. Whereas in principle the liquid crystal is confined by two substrates to a slit-pore geometry we take $s_{z}$ large enough (see Section 4 ) such that a sufficiently big portion of the liquid crystal is unaffected by a direct interaction with both solid substrates (see Section 5.1); the orientation of the mesogens in that bulk-like region is, however, affected indirectly through long-range orientational correlations induced by the solid substrates.

To equilibrate the liquid crystal with the flow being turned off we employ an even simpler model for the mesogen-wall interaction. In this case we smear the discrete solid atoms over the plane of their respective wall. That is we integrate Equation (15) over the position of the solid atoms across the $x-y$ plane and obtain

$$
u_{\mathrm{sw}}\left(\Delta z_{i}^{(k)}\right)=2 \pi \varepsilon_{\mathrm{sw}} n\left[\frac{2}{5}\left(\frac{\sigma}{\Delta z_{i}^{(k)}}\right)^{10}-\left(\frac{\sigma}{\Delta z_{i}^{(k)}}\right)^{4}\right],
$$

where $\varepsilon_{\mathrm{sw}}=\varepsilon_{\mathrm{fs}} / 2 \pi, n=2 / l^{2}$ is the areal density of solid atoms in each wall and $l / \sigma=\sqrt[3]{4}$ is the lattice constant of the fcc (100) plane. In the previous equation, $\Delta z_{i}^{(k)}=$ $\left|z_{i} \pm s_{z} / 2\right|$ is the distance of mesogen $i$ from the upper and lower solid wall, respectively.

\section{Properties}

To analyse our simulations we now introduce a few useful quantities. A central one is the local alignment tensor $\mathbf{Q}$ (see Appendix 2 for its definition from a statistico-mechanical approach). At each point $\boldsymbol{r}, \mathbf{Q}$ is a real, symmetric, traceless, second-rank tensor. It can be represented by a $3 \times 3$ matrix.

As far as our systems of interest are concerned, two different types of alignment tensors $\overline{\mathbf{Q}}$ will be considered that are related to the original $\mathbf{Q}$ through spatial averages. In the first type of situation we have translational symmetry in the $x$ - and $y$-directions. This implies that we can average $\mathbf{Q}$ over these two coordinates according to

$$
\overline{\mathbf{Q}}(z)=\frac{1}{s_{\mathrm{X}} s_{\mathrm{y}}} \int_{-s_{\mathrm{X}} / 2}^{s_{\mathrm{X}} / 2} \mathrm{~d} x \int_{-s_{\mathrm{Y}} / 2}^{s_{\mathrm{y}} / 2} \mathrm{~d} y \mathbf{Q}(\boldsymbol{r}) .
$$

This twofold spatial average is useful in the situation in which there is no flow, the temperature field is homogeneous [i.e. $T_{\text {in }}=T_{\text {out }}$ in Equation (11)], and no optical torque is applied [i.e. $\varepsilon_{\mathrm{opt}}=0$ in Equation (12)].

In the second type of situations when the flow has been turned on (and regardless of whether or not we do have a temperature and a polarising field on top), translational symmetry is preserved only in the $y$-direction. Hence, we can express $\overline{\mathbf{Q}}$ via

$$
\overline{\mathbf{Q}}(x, z)=\frac{1}{s_{\mathrm{y}}} \int_{-s_{\mathrm{y}} / 2}^{s_{\mathrm{y}} / 2} \mathrm{~d} y \mathbf{Q}(\boldsymbol{r}) .
$$

Moreover, we note in passing that in Appendix 2 the local alignment tensor is introduced via ensemble averages assuming that the system under study is at thermodynamic equilibrium. In our NEMD simulations the relevant expressions are, of course, time rather than ensemble averages. In addition, we assume that under steady-state nonequilibrium conditions the equilibrium treatment, from which the expression for $\mathbf{Q}$ is derived, just carries over.

However, regardless of the symmetry of the system to which specific spatial averages of $\overline{\mathbf{Q}}$ correspond, they satisfy an eigenvalue equation of the form

$$
\overline{\mathbf{Q}}(\cdot) \widehat{\boldsymbol{n}}_{0, \pm}(\cdot)=\lambda_{0, \pm}(\cdot) \widehat{\boldsymbol{n}}_{0, \pm}(\cdot),
$$

where $(\cdot)$ is shorthand notation representing either a point along the $z$-axis if $\overline{\mathbf{Q}}$ is taken from Equation (17) or a point in the $x-z$ plane if $\overline{\mathbf{Q}}$ is computed via Equation (18). In Equation (19), $\lambda_{0, \pm}$ are the three eigenvalues and the subscript is shorthand notation referring to the fact that $\lambda_{-} \leq \lambda_{0}<\lambda_{+}$where the equality holds in the absence of biaxiality [see Equation (21) below]; $\widehat{\boldsymbol{n}}_{-}$, $\widehat{\boldsymbol{n}}_{0}$, and $\widehat{\boldsymbol{n}}_{+}$are the corresponding eigenvectors. According to the principles of linear algebra [41] these eigenvectors are pairwise orthogonal to one another because $\overline{\mathbf{Q}}$ is real and symmetric.

Equation (19) is solved numerically using Jacobi's transformation [42]. The alignment tensor can be diagonalised in the basis of its three eigenvectors and may therefore be cast as

$$
\begin{aligned}
\operatorname{diag} \overline{\mathbf{Q}}(\cdot) & =\left(\begin{array}{ccc}
\lambda_{-}(\cdot) & 0 & 0 \\
0 & \lambda_{0}(\cdot) & 0 \\
0 & 0 & \lambda_{+}(\cdot)
\end{array}\right) \\
& =\left(\begin{array}{ccc}
-\frac{S(\cdot)+\xi(\cdot)}{2} & 0 & 0 \\
0 & -\frac{S(\cdot)-\xi(\cdot)}{2} & 0 \\
0 & 0 & S(\cdot)
\end{array}\right),
\end{aligned}
$$

where $S=\lambda_{+}$is the nematic and

$$
\xi(\cdot)=\lambda_{0}(\cdot)-\lambda_{-}(\cdot)
$$

is the biaxiality order parameter. The specific form of $\operatorname{diag} \overline{\mathbf{Q}}$ defining $S$ and $\xi$ is consistent with the phenomenological Landau-de Gennes theory of biaxial nematics (see Chapter 2.3.1 of Ref. [43]). 

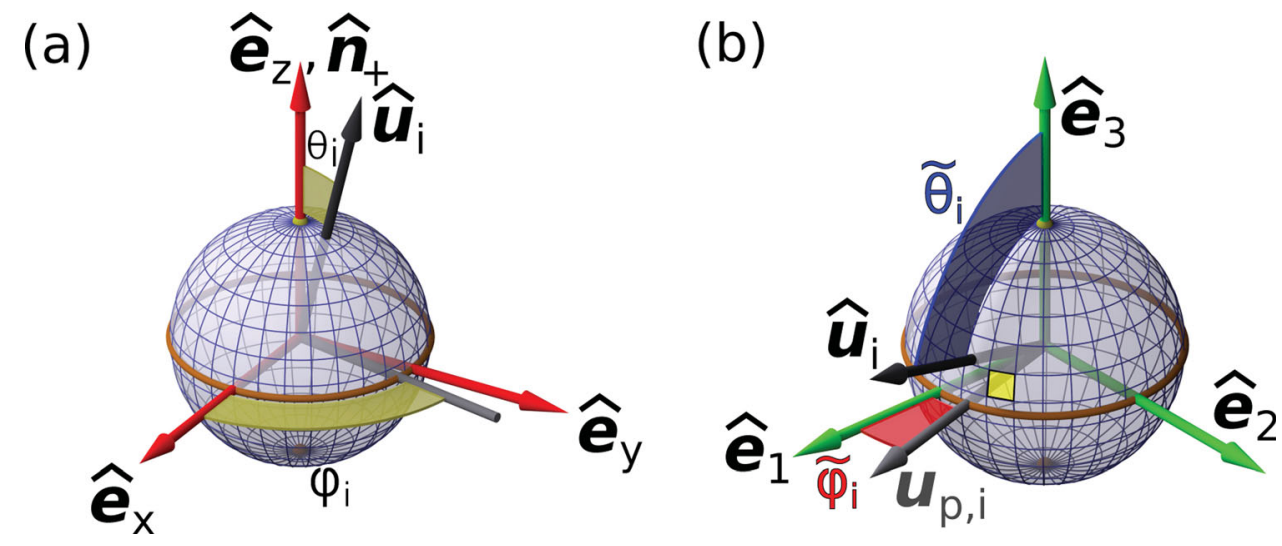

Figure 1. (a) The nematic director $\widehat{\boldsymbol{n}}_{+}$on the unit sphere in the standard basis $\left\{\widehat{\boldsymbol{e}}_{x}, \widehat{\boldsymbol{e}}_{y}, \widehat{\boldsymbol{e}}_{z}\right\}$ where it is assumed that $\widehat{\boldsymbol{n}}_{+} \cdot \widehat{\boldsymbol{e}}_{z}=1$. The unit vector $\widehat{\boldsymbol{u}}_{i}$ [see Equation (2)] specifies the orientation of mesogen $i$. In addition, polar $(\theta)$ and azimuthal $(\varphi)$ angles are also shown. (b) as (a), but for the rotated basis $\left\{\widehat{\boldsymbol{e}}_{1}, \widehat{\boldsymbol{e}}_{2}, \widehat{\boldsymbol{e}}_{3}\right\}$ where $\widetilde{\theta}$ and $\tilde{\varphi}$ are polar and azimuthal angles, respectively. The projection of $\widehat{\boldsymbol{u}}_{i}$ onto the $\widehat{\boldsymbol{e}}_{1}-\widehat{\boldsymbol{e}}_{2}$ plane is given by $\boldsymbol{u}_{\mathrm{p}, i}$. The area shaded in yellow represents a two-dimensional bin of the histogram (see text). The orange circular line marks the equator of the sphere.

Another quantity of interest is the orientation distribution function (odf) defined through the expression

$$
\alpha(x, \widetilde{\omega})=\frac{\sum_{i=1}^{N}\left\langle\delta\left(x-x_{i}\right) \delta\left(\widetilde{\omega}-\widetilde{\omega}_{i}\right)\right\rangle}{\int_{0}^{2 \pi} \int_{0}^{\pi} \sin \widetilde{\theta} \mathrm{d} \widetilde{\theta} \mathrm{d} \widetilde{\varphi}\left\langle\delta\left(x-x_{i}\right) \delta\left(\widetilde{\omega}-\widetilde{\omega}_{i}\right)\right\rangle},
$$

where $\delta$ is the Dirac $\delta$-distribution and $\langle\ldots\rangle$ indicates a time average computed in a stationary nonequilibrium situation (see also Appendix 2).

In Equation (22) the tilde is used to signal that in order to compute the odf in a simulation it is numerically advantageous to express the orientations in a coordinate system that has been rotated with respect to the original one spanned by the standard basis. This is because in a computer simulation, $\alpha$ is obtained as a two-dimensional histogram. To that end, one partitions the surface of a sphere into small bins of area $\delta \widetilde{A}=\sin \widetilde{\theta} \delta \widetilde{\theta} \delta \widetilde{\varphi}$ and simply counts the number of mesogens with an orientation pertaining to a specific bin.

As indicated by the sketch in Figure 1, the area $\delta \widetilde{A}$ of a bin depends on its location on the surface of the sphere: $\delta \widetilde{A}$ is largest for bins situated along the equator of the sphere but gradually vanishes as one approaches one of the poles. Thus, from a statistics point of view it would be advantageous to replace the standard basis by a new one $\left\{\widehat{\boldsymbol{e}}_{1}, \widehat{\boldsymbol{e}}_{2}, \widehat{\boldsymbol{e}}_{3}\right\}$ that is rotated with respect to the standard basis. If this passive rotation is carried out properly a unit vector $\widehat{\boldsymbol{u}}_{i}$ will always be sorted into the largest possible bins. This way the odf is calculated with maximum statistical accuracy and resolution.

Let us consider a situation, on the one hand, in which the mesogens are aligned homeotropically at the plane of the solid walls. If this alignment is perfect it complies with $\widehat{\boldsymbol{n}}_{+} \cdot \widehat{\boldsymbol{e}}_{\mathrm{z}}=1$ [see Figure 1(a)]. If, on the other hand, the mesogens are exposed to the optical torque we have $\widehat{\boldsymbol{n}}_{+}$. $\widehat{\boldsymbol{e}}_{\mathrm{y}}=1$ if the orientation of the mesogens is again ideal because of Equation (14).

Hence, we want to carry out a passive rotation such that the equations $\widehat{\boldsymbol{e}}_{1}=\mathbf{R} \widehat{\boldsymbol{e}}_{\mathrm{x}}=\widehat{\boldsymbol{e}}_{\mathrm{y}}$ and $\widehat{\boldsymbol{e}}_{2}=\mathbf{R} \widehat{\boldsymbol{e}}_{\mathrm{y}}=\widehat{\boldsymbol{e}}_{\mathrm{z}}$ are satisfied. Because the basis $\left\{\widehat{\boldsymbol{e}}_{1}, \widehat{\boldsymbol{e}}_{2}, \widehat{\boldsymbol{e}}_{3}\right\}$ should consist of unit vectors that are pairwise orthogonal, $\widehat{\boldsymbol{e}}_{3}=\mathbf{R} \widehat{\boldsymbol{e}}_{\mathrm{z}}=\widehat{\boldsymbol{e}}_{\mathrm{x}}$ must also hold. From these three equations one easily sees that

$$
\mathbf{R}=\left(\begin{array}{lll}
0 & 0 & 1 \\
1 & 0 & 0 \\
0 & 1 & 0
\end{array}\right)
$$

The angles $\widetilde{\theta}_{i}$ and $\widetilde{\varphi}_{i}$ in Figure 1(b) can be computed by actively rotating $\widehat{\boldsymbol{u}}_{i}$ [see Equation (2)] according to

$$
\begin{aligned}
\widehat{\boldsymbol{u}}\left(\widetilde{\omega}_{i}\right) & =\left(\begin{array}{c}
\sin \widetilde{\theta}_{i} \cos \widetilde{\varphi}_{i} \\
\sin \widetilde{\theta}_{i} \sin \widetilde{\varphi}_{i} \\
\cos \widetilde{\theta}_{i}
\end{array}\right) \\
& =\mathbf{R}^{-1} \widehat{\boldsymbol{u}}\left(\omega_{i}\right)
\end{aligned}
$$

for $i=1, \ldots, N$. We can easily solve Equation (24) for $\widetilde{\theta}_{i}$ and $\widetilde{\varphi}_{i}$ and then compute the odf from Equation (22).

An additional benefit of using the rotated coordinate system is that it helps to simplify the analysis of the odf. Clearly, if a portion of the liquid crystal becomes polar on account of $\widehat{\boldsymbol{p}}$, one expects the odf to exhibit a single maximum centred on $\widetilde{\varphi}=0$ and $\widetilde{\theta}=\pi / 2$. If, on the other hand, the nematic phase possesses head-tail symmetry (that is, $\widehat{\boldsymbol{n}}_{+}$and $-\widehat{\boldsymbol{n}}_{+}$are equivalent), one anticipates the odf to have two instead of just a single maximum. One of these is centred on $\widetilde{\varphi}=-\pi / 2$ and $\widetilde{\theta}=\pi / 2$. The second maximum is also centred at $\widetilde{\theta}=\pi / 2$ but shifted to $\tilde{\varphi}=\pi / 2$.

With the odf we can compute the local polarisation of the liquid crystal which arises in the direction of the flow 
on account of the optical torque $\boldsymbol{\tau}_{\text {opt }}$ [see Equation (13)]. Following earlier work $[44,45]$ we focus on components of the local polarisation vector $\boldsymbol{P}$ which we introduce via [cf., Equation (24)]

$$
\boldsymbol{P}(x)=\int \mathrm{d} \widetilde{\omega} \widehat{\boldsymbol{u}}(\widetilde{\omega}) \alpha(x, \widetilde{\omega}) .
$$

With the aid of Equations (A.62) and (A.63) of Ref. [32] we can compute (Cartesian) components of $\boldsymbol{P}$. For example, it is easy to realise that

$$
\begin{aligned}
P_{\mathrm{x}}(x)= & \int \mathrm{d} \widetilde{\omega} \alpha(x, \widetilde{\omega}) \sin \widetilde{\theta} \cos \widetilde{\varphi} \\
= & -\sqrt{\frac{4 \pi}{3}}\left(\frac{1}{2}\right)^{1 / 2} \int \mathrm{d} \widetilde{\omega} \alpha(x, \widetilde{\omega}) \\
& \times\left[\mathcal{Y}_{11}(\widetilde{\omega})+\mathcal{Y}_{11}^{*}(\widetilde{\omega})\right] \\
= & -\sqrt{\frac{8 \pi}{3}} \int \mathrm{d} \widetilde{\omega} \alpha(x, \widetilde{\omega}) \operatorname{Re} \mathcal{Y}_{11}(\widetilde{\omega}) .
\end{aligned}
$$

Similarly, we obtain

$$
\begin{aligned}
& P_{\mathrm{y}}(x)=\sqrt{\frac{8 \pi}{3}} \int \mathrm{d} \widetilde{\omega} \operatorname{Im} \mathcal{Y}_{11}(\tilde{\omega}) \alpha(x, \widetilde{\omega}) \\
& P_{\mathrm{Z}}(x)=\sqrt{\frac{4 \pi}{3}} \int \mathrm{d} \widetilde{\omega} \mathcal{Y}_{10}(\widetilde{\omega}) \alpha(x, \widetilde{\omega}) .
\end{aligned}
$$

In Equations (26) and (27a), 'Re' and 'Im' denote real and imaginary parts, respectively. From Equations (26)-(27b) it is straightforward to compute the polarisation

$$
\begin{aligned}
P(x) & =|\boldsymbol{P}(x)| \\
& =\sqrt{P_{\mathrm{x}}^{2}(x)+P_{\mathrm{y}}^{2}(x)+P_{\mathrm{z}}^{2}(x)}
\end{aligned}
$$

which we will also be considering below.

To make sure that under the conditions of our NEMD simulations a laminar flow is eventually established we compute the Reynolds number defined as [46]

$$
\mathcal{R}=\frac{\bar{\rho} v_{\infty} \ell}{\eta}
$$

which expresses the ratio of inertial to viscous forces. In Equation (29), $\bar{\rho}$ is the mean density, $v_{\infty}$ is the maximum of the steady-state flow velocity in the $x$-direction (i.e. in the direction of the flow), $\ell$ is a characteristic length, and $\eta$ is the dynamic viscosity. The physical significance of $\ell$ is admittedly somewhat ambiguous. Because the velocity profile is established perpendicular to the solid substrates and following previous work [39], we assume $\ell=s_{\mathrm{z}}$.

For our present setup we expect Poiseuille flow on account of the atomically structured solid substrates [see Equation (15)]. Poiseuille flow is characterised by a parabolic velocity profile given by the expression

$$
v_{\mathrm{x}}(z)=\frac{F_{\mathrm{e}} \bar{\rho}}{2 \eta}\left(z^{2}-\frac{s_{\mathrm{z}}^{2}}{4}\right),
$$

where $v_{\mathrm{X}}=\boldsymbol{v} \cdot \widehat{\boldsymbol{e}}_{\mathrm{X}}$ is the $x$-component of the velocity field $\boldsymbol{v}$. Equation (30) can be derived from the linearised Navier-Stokes equation invoking different approximations [36,39,47-49]. Fitting Equation (30) to velocity profiles generated in the NEMD simulations permits one to determine $\eta[36,39]$ and with it $\mathcal{R}$ from Equation (29). The reader should appreciate that Equation (30) is valid in the absence of the optical torque [see Equation (13)] and if the temperature $T_{0}$ is constant everywhere in the system [see Equation (8)].

\section{Numerical details}

In the following we will give all physical quantities in dimensionless (i.e. 'reduced') units. In our NEMD simulations, we take as basic units the mass $m$ of a mesogen, the diameter $\sigma$ of its spherical core, and the depth $\varepsilon$ of the attractive well of $u_{\mathrm{LJ}}$ [see Equation (A6)]. Other dimensionless units are defined via suitable combinations of these basic ones (see Appendix B of the book by Allen and Tildesley [50]).

To integrate the equations of motion we employ the velocity Verlet algorithm as suggested by Ilnytskyi and Wilson for Lennard-Jones and Gay-Berne molecules [51] with a time step $\delta t=10^{-3}$. Interactions between mesogens are cut off if their distance exceeds $r_{\mathrm{c}}=3.0$. We typically place $N \approx 15,600$ mesogens in a simulation cell with side lengths of $s_{\mathrm{X}}=100.0, s_{\mathrm{y}}=8.0-9.0$, and $s_{\mathrm{z}}=$ 24.0 in the $x$-, $y$-, and $z$-directions, respectively. To save computer time we partition the simulation cell into small boxes using a linked-cell neighbour list [50]. A given box is considered a neighbour of its neighbouring boxes if the distance between their centres does not exceed 6.0.

Because we are interested in relatively low flow speeds we need to equilibrate the system for a reasonably large number of time steps ranging from $10^{5}$ to $2.0 \times 10^{5}$. Equilibration proceeds in two steps. First, we use a simulation in the isothermal-isobaric ensemble in the absence of flow and with the laser turned off. This is followed by a second equilibration in the same ensemble with the laser being turned on and eventually the flow is turned on as well.

Once a steady-state nonequilibrium situation has been reached, averages are collected for another $2.0 \times 10^{6}$ time steps. Equilibration runs are performed in the isothermal-isobaric ensemble by fixing the pressure (tensor component) in the $y$-direction to $P=1.8$. That way and because of the size of the system we generate a 
bulk-like region of a typical liquid density as we shall be demonstrating below. Taking the average side length $s_{y}$ in that direction at the end of the equilibration run as input, the production run is then performed in the canonical ensemble.

In both, the equilibration and the averaging part of the simulation the body force $F_{\mathrm{e}}$ is applied in the $x$-direction in a small cuboid of volume $\delta s_{\mathrm{X}} \times s_{\mathrm{y}} \times s_{\mathrm{Z}}$ where $\delta s_{\mathrm{X}}=$ 2.0; the cuboid begins at the left end of the simulation box at $x=-s_{\mathrm{x}} / 2$.

\section{Results}

\subsection{Structure of the nematic liquid crystal at thermodynamic equilibrium}

We begin the discussion of our results by demonstrating that our system is sufficiently large to avoid spurious effects resulting from the wall-induced stratification of the liquid crystal. To that end we introduce the average local density

$$
\rho(z)=\frac{\langle N(z)\rangle}{A \delta z},
$$

where $\langle N\rangle$ is the average number of mesogens having their centres of mass located in a small interval of size $\delta z$ centred on $z ; A$ is the area of planes normal to the $z$ axis of the Cartesian coordinate system (i.e. parallel to the planes of the walls).

Equation (31) tacitly assumes that the local density depends only on $z$. Strictly speaking, this is not the case on account of the atomistic corrugation of the solid walls reflected by the dependence of $u_{\mathrm{fs}}$ on $r_{i j}$. Therefore, Equation (31) has to be viewed as an approximation.

The plot in Figure 2(a) shows that in the immediate vicinity of the wall atoms $\rho=0$ on account of strongly repulsive interactions between the mesogens and the solid walls. As one moves away from the solid walls, $\rho$ oscillates with decreasing $|z|$ which reflects stratification, that is the packing of the mesogens in individual layers. However, the amplitude of the oscillations decreases quickly and is essentially zero for $|z| \lesssim 5$.0. This reflects the short-range character of $u_{\mathrm{fs}}$. Therefore, a bulk-like region exists for $|z| \lesssim 5.0$.

A plot of the corresponding nematic order parameter $S$ is presented in Figure 2(b). In the immediate vicinity of the solid walls, $S=0$ for the same reasons already explained. As $|z|$ decreases, $S$ decays and exhibits weak and rapidly damped oscillations. They reflect the same layering phenomenon that was seen in the plot of $\rho$ in Figure 2(a).

Below $|z| \simeq 5.0$ we concluded from Figure 2(a) that a bulk-like region exists characterised by a constant density of $\rho \simeq 0.85$. In this so defined bulk-like regime the plot in

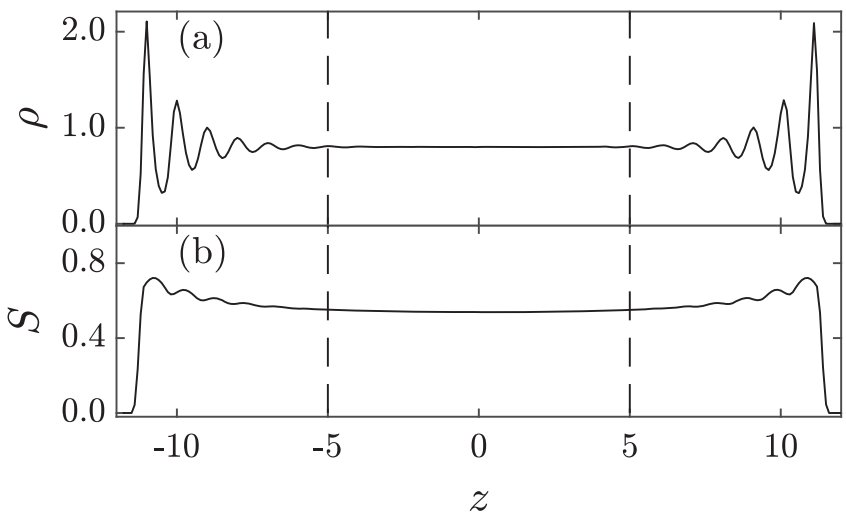

Figure 2. (a) Plot of the average local density $\rho$ as a function of the position $z$ between the solid walls located at $z= \pm s_{z} / 2=$ \pm 24 .0. The dashed vertical lines demarcate an essentially bulklike region centred on $z=0$ in which the constant bulk density $\rho \simeq 0.85$ is attained. Data have been obtained for $N \simeq 15,600$ mesogens and a temperature of $T=0.90$; (b) as (a), but for the nematic order parameter $S$.

Figure 2(b) reveals that $S$ decreases slightly until it reaches its minimum of $S \simeq 0.54$ at $z=0$.

However, the reader should realise that this value is still indicative of the existence of a nematic phase. For example, if one applies Landau-de Gennes theory a stable nematic phase is expected to form at a transition temperature below which $S \geq \frac{1}{3}$ [52]. A more sophisticated computer simulation based finite-size scaling approach would suggest that for the present model the threshold value is $S \simeq 0.36$ [52] which is surprisingly close to the Landau-de Gennes result.

\subsection{The impact of external fields in the absence of flow}

The focus of this work is ultimately on the combined effects of flow, local heating, and an optical torque on the structure of a nematic liquid crystal. As expected the local heating gives rise to a temperature gradient in the $x$-direction as one can see from the plots in Figure 3(a). The temperature profile obtained from the simulation follows very closely the one imposed by Equation (11) indicating that the Galilean thermostat represented by Equation (8) gives physically meaningful results free from any spurious effects.

We also plot in Figure 3(b) the total polarisation computed from Equation (28) for various coupling strengths $\varepsilon_{\text {opt }}$ of the optical torque [see Equation (13)]. For sufficiently weak coupling, $P$ exhibits a nearly Gaussian shape as dictated by Equation (13); however, at increasingly higher values of $\varepsilon_{\mathrm{opt}}$ a plateau forms around the centre of the area in which $\boldsymbol{\tau}_{\text {opt }}$ acts upon the mesogens. 


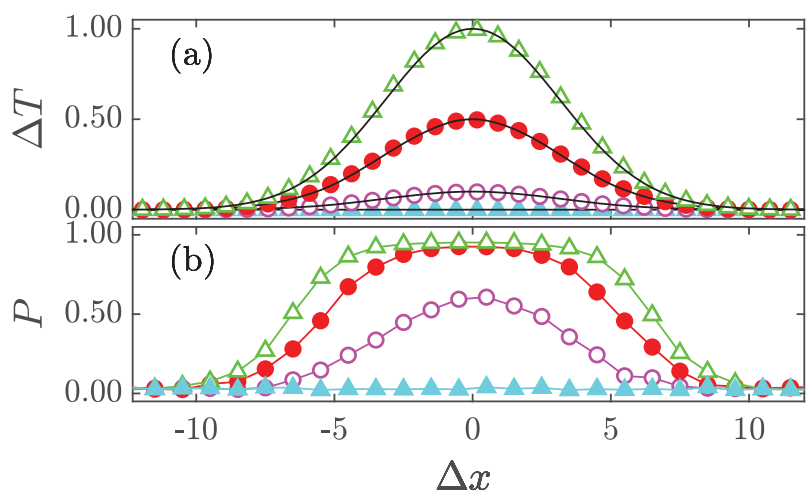

Figure 3. Nematic liquid crystal exposed to both thermal heating and polarisation due to the laser. (a) Plots of the local change in temperature $\Delta T(\Delta x)=T_{0}^{\prime}-T_{0}(\Delta x)\left[\Delta x=x-x_{0}\right.$, see Equations (8) and (11)]; ( $\Delta) T_{\text {in }}=0.9,(0) T_{\text {in }}=1.0,(\bullet) T_{\text {in }}=1.4,(\Delta)$ $T_{\text {in }}=1.9$. In all cases $T_{\text {out }}=T_{0}=0.9$ and $\varepsilon_{\text {opt }}=0.0$. Solid lines represent Equation (11) at the corresponding temperatures. (b) As (a), but for the polarisation $P$ [see Equation (28)] where $(\Delta)$ $\varepsilon_{\text {opt }}=0.0,(\circ) \varepsilon_{\text {opt }}=5.0,(\bullet) \varepsilon_{\text {opt }}=15.0,(\Delta) \varepsilon_{\text {opt }}=30.0$. The solid lines represent fits intended to guide the eye. The plots have been obtained for $T_{\text {in }}=T_{\text {out }}=0.9$.

According to one's physical intuition one anticipates that a (local) increase in the temperature reduces the order upon increasing temperature. However, the joint effect of flow plus an optical torque is a bit more difficult to disentangle. It therefore seems prudent to first consider the impact of the optical torque in a system at thermodynamic equilibrium [without local heating and in the absence of flow, that is for $T_{\text {in }}=T_{\text {out }}$ in Equation (11) and for $F_{\mathrm{e}}=0$ ].

Plots in Figure 4 illustrate the impact of $\boldsymbol{\tau}_{\text {opt }}$ alone in greater detail. Because of the underlying Gaussian in our definition of $\boldsymbol{\tau}_{\text {opt }}$ in Equation (13) we define as the width $\delta x$ of the central region in which the optical torque is appreciable, two times the confidence interval of the Gaussian profile in Equation (13). Under the conditions chosen in this study, $\delta x \simeq 12.0$.

The plot in Figure 4(a) reveals that sufficiently far away from the central region [i.e. for $|\Delta x| \gtrsim 20.0$ ] the local nematic order parameter $S \simeq 0.54$. This value does not vary much with position and the associated director field is fairly homogeneous pointing in the $z$-direction. The latter is a consequence of the homeotropic alignment of the mesogens at the solid walls. In this regime, $\widehat{\boldsymbol{n}}_{+} \simeq n_{\mathrm{x},+} \widehat{\boldsymbol{e}}_{\mathrm{x}}+n_{\mathrm{z},+} \widehat{\boldsymbol{e}}_{\mathrm{z}}$ and $n_{\mathrm{x},+}^{2}+n_{\mathrm{z},+}^{2} \simeq 1$ as well as $\left|n_{\mathrm{y},+}\right| \simeq 0$.

As one approaches the central region illuminated by the laser beam, two effects can be seen in Figure 4(a). First, $S$ decreases considerably and second, the small dashes representing $\widehat{\boldsymbol{n}}_{+}$eventually shrink in length. The shrinkage of $\widehat{\boldsymbol{n}}_{+}$is relatively easy to understand. Because $\left|\widehat{\boldsymbol{n}}_{+}\right|=1$ regardless of its orientation in space, the dashes

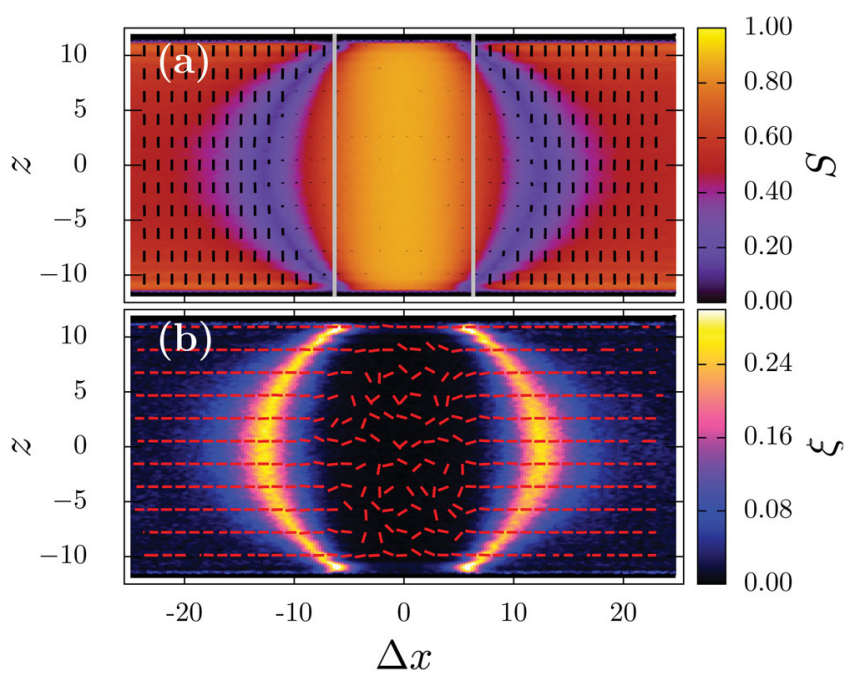

Figure 4. Nematic liquid crystal exposed only to polarisation due to the laser. (a) Plot of the local nematic order parameter $S(x, z)$ [see Equation (18)] in the $x-z$ plane as a function of the distance $\Delta x=x-x_{0}$ from the centre of the external field at $x_{0}$; the magnitude of $S(x, z)$ is given by the bar attached. The direction of the associated director field $\widehat{\boldsymbol{n}}(\boldsymbol{r})$ is represented by the short dashes (see text). The distance between the two long vertical lines corresponds to two times the confidence interval of the Gaussian in Equation (13). (b) As (a), but for the local biaxiality order parameter $\xi(x, z)$ [see Equation (21). In this case, the dashes represent $\widehat{\boldsymbol{n}}_{-}(\boldsymbol{r})$. Data have been obtained at thermodynamic equilibrium $\left(F_{\mathrm{e}}=0\right)$ without local heating $\left[T_{\text {in }}=T_{\text {out }}=0.90\right.$, see Equation (11) and Figure $3(\mathrm{a})]$ and a polarising field $\left[\varepsilon_{\mathrm{opt}}=15.0\right.$, see Equation (13)].

representing the nematic director become shorter if the net orientation of the mesogens is rotated out of the $x-z$ plane because then $n_{\mathrm{x},+}^{2}+n_{\mathrm{z},+}^{2}<1$. Hence, the shorter dashes visible near the central region of our system indicate that $\widehat{\boldsymbol{n}}_{+}$aligns increasingly with the $y$ axis. Notice, that in the ideal case of perfect alignment the dashes vanish completely as then $n_{\mathrm{x},+}^{2}+n_{\mathrm{z},+}^{2}=0$. The latter is observed directly inside the central region where, on account of $\boldsymbol{\tau}_{\text {opt }}, S$ increases to a maximum of about 0.95 .

Thus, the process illustrated in Figure 4(a) is the following. Sufficiently far from the laser beam (i.e. for, say, $\Delta x \lesssim-20.00)$ the preferred alignment of the mesogens is homeotropic with respect to the plane of the solid walls because the mesogens are slightly elongated. This is because in this region $u_{\mathrm{fs}}$ dominates the orientational order [see Equation (15)]. This order is long-range despite the short-range character of $u_{\mathrm{fs}}$ [see Figure 2(b)].

As one moves into the region illuminated by the laser beam, the increasing optical torque then rotates the longer axes of the mesogens. Eventually the mesogens are nearly perfectly aligned with the $y$-axis in the central region (i.e. for $|\Delta x| \lesssim 6.0$ ). Moving then out of the central region (i.e. for $\Delta x \gtrsim 6.0$ ) the mesogens are 
rotated back to the $x-z$ plane because the mesogenwall interaction begins to dominate the orientational structure again. Eventually the mesogens assume their original homeotropic alignment at the solid walls; the homeotropic alignment is then transferred into regions further away from the walls (i.e. beyond the range of $u_{\mathrm{fs}}$ ) through a cooperative, long-range mechanism.

It is now instructive to correlate the plots of $S$ and $\widehat{\boldsymbol{n}}_{+}$ in Figure 4(a) with those of the biaxiality order parameter $\xi$ and $\widehat{\boldsymbol{n}}_{-}$in Figure 4(b). From the plot of $\xi$ one can see that there is quite a bit of biaxial order in regions in which $S$ in Figure 4(a) decreases. We refer to these regions as the nematic and biaxiality halo, respectively, as they surround the laser beam. The origin of this biaxiality can be rationalised as follows.

The three eigenvectors of $\overline{\mathbf{Q}}$ form a set of orthonormal basis vectors so that $\widehat{\boldsymbol{n}}_{+} \cdot \widehat{\boldsymbol{n}}_{-}=0$ everywhere in the system. Consider then the plot of $\widehat{\boldsymbol{n}}_{-}$in Figure 4(b). Until one reaches the region in which $\boldsymbol{\tau}_{\text {opt }}$ becomes appreciable (i.e. for $|\Delta x| \gtrsim 6.0$ ), $\widehat{\boldsymbol{n}}_{-}$is parallel to the $x$-axis. Moreover, we already saw from the plot of $\widehat{\boldsymbol{n}}_{+}$in Figure 4(a) that as one approaches the central region of $|\Delta x| \lesssim 6.0$, the nematic director begins to rotate out of the $x-z$ plane and aligns increasingly with $\widehat{\boldsymbol{e}}_{\mathrm{y}}$. The axis of this rotation is $\widehat{\boldsymbol{e}}_{\mathrm{x}}$.

Thus, when this happens $\widehat{\boldsymbol{n}}_{-}$changes its physical significance from just being one of the three (pairwise orthonormal) eigenvectors of $\overline{\mathbf{Q}}$ to the common axis of active rotation for the set of mesogenic orientations $\left\{\widehat{\boldsymbol{u}}_{i}\right\}$ (and therefore for $\widehat{\boldsymbol{n}}_{+}$). Thus, we now have two distinct symmetry axes, namely $\widehat{\boldsymbol{n}}_{+}$and the axis of rotation $\widehat{\boldsymbol{e}}_{\mathrm{x}}=$ $\widehat{\boldsymbol{n}}_{-}$. This is the reason for the existence of the biaxial halo. According to the definition of $\xi$ in Equation (21) the biaxial halo is that region of the liquid crystal in which the laser-induced optical torque abrogates the degeneracy of the eigenvalues $\lambda_{-}$and $\lambda_{0}$.

The plot of $\widehat{\boldsymbol{n}}_{-}$also reveals that it remains approximately parallel to the $x$-axis until the central region of the laser beam is approached (i.e. until $|\Delta x| \simeq 6$.0. In this central region the mesogens are almost perfectly aligned with the $y$-axis and $\widehat{\boldsymbol{n}}_{+}$is hardly visible anymore for reasons already explained. In the central region, $\widehat{\boldsymbol{n}}_{-}$exhibits two characteristic features.

First, locally $\widehat{\boldsymbol{n}}_{-}$is no longer aligned with the $x$-axis and second the short dashes representing $\widehat{\boldsymbol{n}}_{-}$are more or less of the same length. This indicates that locally and regardless of the specific position the eigenvectors $\widehat{\boldsymbol{n}}_{-}$are restricted to the $x-z$ plane with an infinitely large degeneracy of their orientation. This is because $\widehat{\boldsymbol{n}}_{-}$must always be orthogonal to $\widehat{\boldsymbol{n}}_{+}$and because $\widehat{\boldsymbol{n}}_{+}$is nearly perfectly aligned with the $y$-axis on account of a sufficiently strong optical torque. However, there is no mechanism discriminating any specific orientation of $\widehat{\boldsymbol{n}}_{-}$in the $x-z$ plane as

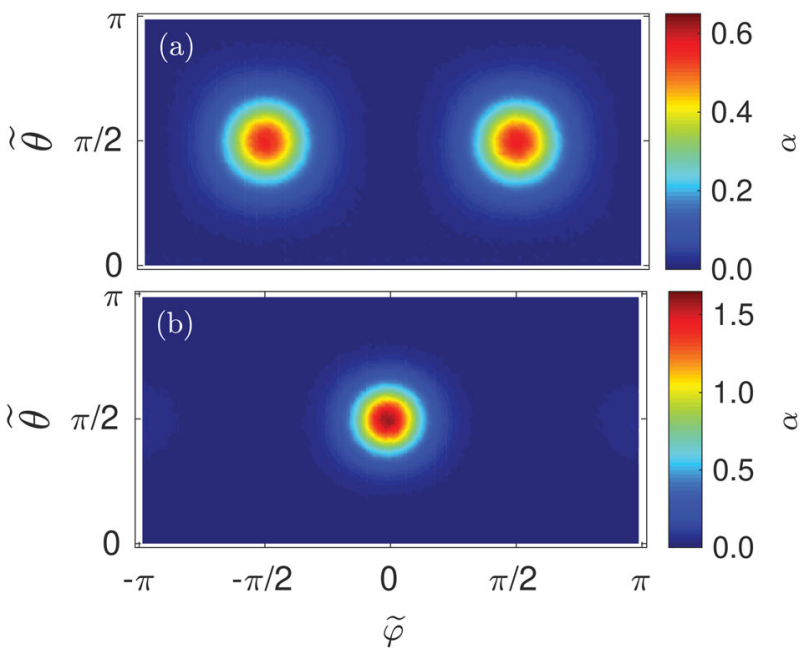

Figure 5. Plots of the orientation distribution function $\alpha(x, \widetilde{\omega})$ in the $\widetilde{\varphi}-\widetilde{\theta}$ plane [see Figure 1]. The height of $\alpha(x, \widetilde{\omega})$ can be read off the bars attached to the plots on the right where we also emphasise the different scales used. Part (a) corresponds to $x$ sufficiently far away from the laser beam whereas part (b) of the figure corresponds to $x=x_{0}$ (i.e. $\Delta x=0$ in Figure 4). Notice, that in both parts of the figure, $\alpha(x, \widetilde{\omega})$ has been averaged over a sufficiently wide region centred on $x$ in which the plot of $S(x, z)$ indicates a more or less homogeneously ordered nematic phase. In both parts of the figure $T_{\text {in }}=1.0, T_{\text {out }}=0.9$, and $\varepsilon_{\text {opt }}=15.0$ [see Equations (11), (13), and (14)].

long as the orthogonality of $\widehat{\boldsymbol{n}}_{-}$and $\widehat{\boldsymbol{n}}_{+}$is preserved (i.e. as long as $\widehat{\boldsymbol{n}}_{-} \cdot \widehat{\boldsymbol{e}}_{\mathrm{y}}=0$ is satisfied).

The orientational features discussed so far are consistent with the changes in the structure of the odf plotted in Figure 5. As one can see, the odf in Figure 5(a) exhibits two relatively localised peaks centred on $\widetilde{\varphi}= \pm \pi / 2$ and $\widetilde{\theta}=\pi / 2$. To rationalise these angles let us assume that $\widehat{\boldsymbol{n}}_{+} \cdot \widehat{\boldsymbol{e}}_{\mathrm{z}}=1$ (homeotropic alignment). Thus, we have

$$
\begin{aligned}
\widehat{\boldsymbol{n}}_{+}(\widetilde{\omega}) & =\mathbf{R}^{-1} \widehat{\boldsymbol{n}}_{+}(\omega)=\left(\begin{array}{c}
\sin \tilde{\theta} \cos \widetilde{\varphi} \\
\sin \widetilde{\theta} \sin \widetilde{\varphi} \\
\cos \widetilde{\theta}
\end{array}\right) \\
& =\mathbf{R}^{-1} \widehat{\boldsymbol{e}}_{\mathrm{z}}=\left(\begin{array}{l}
0 \\
1 \\
0
\end{array}\right),
\end{aligned}
$$

where the inverse rotation matrix $\mathbf{R}^{-1}$ can easily be obtained from Equation (23). Equation (32) therefore implies $\widetilde{\varphi}=\widetilde{\theta}=\pi / 2$. Noticing also that $\widehat{\boldsymbol{n}}_{+}$and $-\widehat{\boldsymbol{n}}_{+}$ describe the same physics, $\widetilde{\varphi}=-(\pi / 2)$ would also be permissible in Equation (32). Thus, one would anticipate the odf to have maxima at $\widetilde{\varphi}= \pm \pi / 2$ and for $\widetilde{\theta}=\pi / 2$ and this is what is indeed observed in Figure 5(a). Moreover, the two maxima visible in the plot in Figure 5(a) have equal heights thus supporting the equivalence of $\widehat{\boldsymbol{n}}_{+}$and $-\widehat{\boldsymbol{n}}_{+}$. We therefore conclude that the plot shown 
in Figure 5(a) corresponds to a 'normal' (i.e. nonpolar) nematic phase.

Let us now assume that $\widehat{\boldsymbol{n}}_{+}$is aligned with the $y$-axis, that is $\widehat{\boldsymbol{n}}_{+} \cdot \widehat{\boldsymbol{e}}_{\mathrm{y}}=1$. In this case

$$
\widehat{\boldsymbol{n}}_{+}(\widetilde{\omega})=\mathbf{R}^{-1} \widehat{\boldsymbol{n}}_{+}(\omega)=\left(\begin{array}{l}
1 \\
0 \\
0
\end{array}\right)
$$

consistent with $\widetilde{\varphi}=0$ and $\widetilde{\theta}=\pi / 2$. An inspection of the plot in Figure 5(b) shows that this agrees with the odf presented in that figure. As one can also see the odf has only a single maximum. In other words, $\widehat{\boldsymbol{n}}_{+}$and $-\widehat{\boldsymbol{n}}_{+}$are not equivalent and the nematic phase represented by the odf in Figure 5 must be polar where the direction of the polarisation is in the direction of $\widehat{\boldsymbol{e}}_{\mathrm{y}}$. This is consistent with $\boldsymbol{\tau}_{\text {opt }}$ specified in Equations (13) and (14). Notice also that the maximum of the odf plotted in Figure 5(b) exceeds the ones in Figure 5(a) by a factor of 2.5-3.0 reflecting the relative degree of order inside and outside of the laser beam.

\subsection{The impact of flow}

Having characterised the structure of the liquid crystal in the absence of flow, we now turn to its nonequilibrium behaviour at steady-state flow conditions. We begin by focusing on the behaviour of the nematic liquid crystal under flow but in the absence of any other external field, that is we set $T_{\mathrm{in}}=T_{\mathrm{out}}$ in Equation (11) and $\varepsilon_{\mathrm{opt}}=0$ in Equation (13).

We intend to limit ourselves to laminar-flow scenarios, that is to flow which can be described in terms of individual fluid layers moving at different relative speeds [53] in the direction of the external body force $F_{\mathrm{e}}$. Because $F_{\mathrm{e}}$ acts in the $x$-direction we focus on the velocity profile in that same direction and as a function of the position $z$ between the solid walls.

Plots of $v_{\mathrm{x}}$ are presented in Figure 6 for various strengths of $F_{\mathrm{e}}$. In general, and irrespective of the magnitude of $F_{\mathrm{e}}$, parabolic velocity profiles are observed. The shape of the profiles is therefore consistent with Equation (30) that was obtained as a solution of the linearised Navier-Stokes equation.

Towards the solid walls (i.e. as $|z| \rightarrow 12.5$ ), $v_{\mathrm{x}}$ decays monotonically and then vanishes in the immediate vicinity of the walls where the corresponding plot of $\rho$ in Figure 2(a) reveals that the local density drops to zero as well. These general features are to be expected for true Poiseuille flow $[48,49]$.

If, however, $F_{\mathrm{e}}$ exceeds a certain (model dependent) threshold value, the parabolic shape of $v_{\mathrm{x}}$ is maintained at first but the curves do no longer drop to zero as one

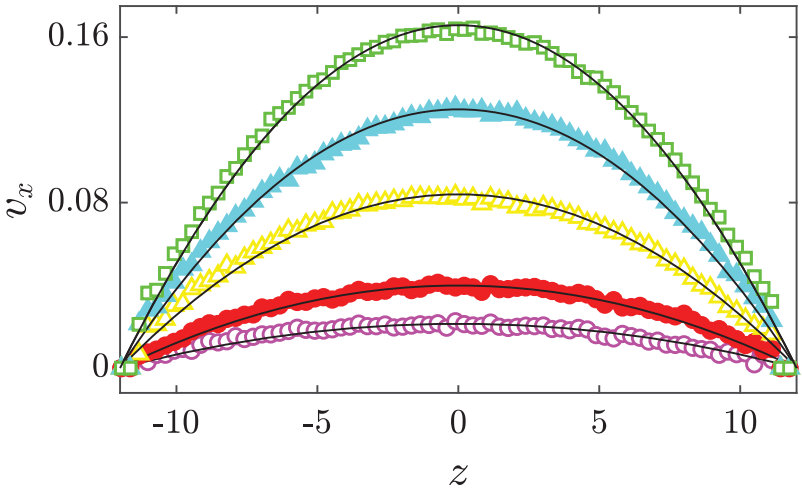

Figure 6. As Figure 2(a), but for the $x$-component the velocity field $\boldsymbol{v} ;(\bigcirc) F_{\mathrm{e}}=0.05,(\circlearrowleft) F_{\mathrm{e}}=0.10,(\Delta) F_{\mathrm{e}}=0.20,(\Delta) F_{\mathrm{e}}=0.30$, $(\square) F_{\mathrm{e}}=0.40$. Solid lines are fits of Equation (30) to the discrete data points.

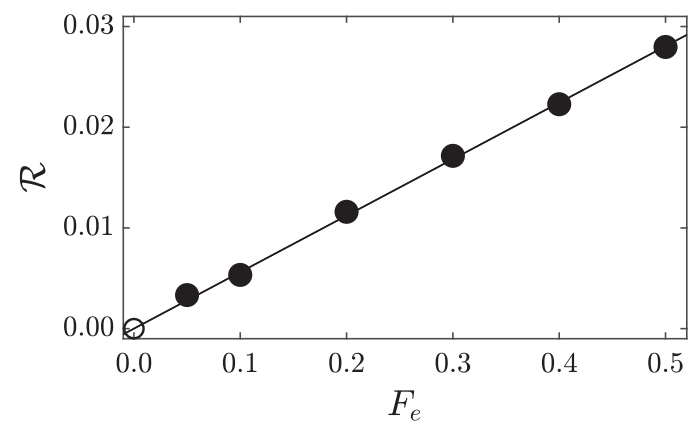

Figure 7. Plot of the Reynolds number $\mathcal{R}$ as a function of the strength of the external body force $F_{\mathrm{e}}$ where $(\bullet)$ is computed from the NEMD data using Equation (29) (see text). The solid line is a fit to the discrete data points intended to guide the eye. The point for $F_{\mathrm{e}}=0(\mathrm{o})$ is included to demonstrate the consistency of the data obtained from the NEMD simulations.

approaches the walls indicating that for these values of the body force the friction exerted by the wall atoms is insufficient [see, for example, Figure 2 of Ref. [39]]. Unfortunately, the strength of the interaction between a mesogen and a wall atom cannot be made much larger before the portion of the liquid crystal closest to the wall forms an unwanted glassy structure.

From the fit of Equation (30) to the discrete data points plotted in Figure 6 we compute the dynamic viscosity $\eta$ which we are treating as a fit parameter. Using this latter quantity we compute the Reynolds number $\mathcal{R}$ that characterises the flow regime we are in. Specifically, one can see from the plot in Figure 7 that over the entire range of body forces $F_{\mathrm{e}}$ considered, $\mathcal{R} \ll 1$. This implies that under the conditions chosen in this work we are in the Stokes (or creeping) flow regime, where viscous forces surpass the inertial ones.

Turning on the flow has remarkable consequences for the local nematic order parameter $S$, the local biaxiality order parameter $\xi$, as well as for the orientation of the 

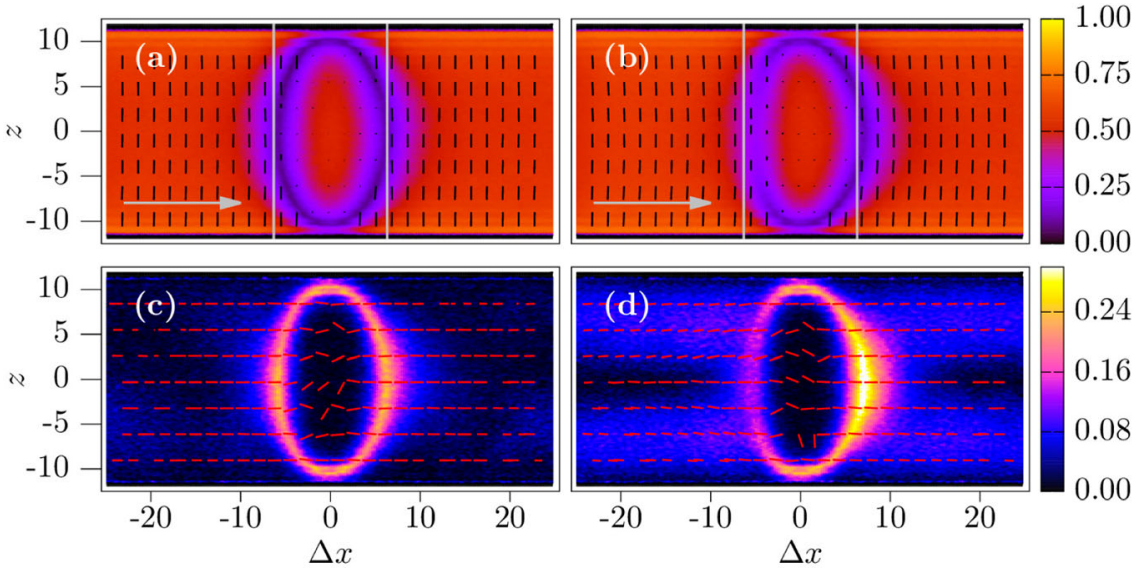

Figure 8. As Figure 4, but in the presence of flow for $F_{\mathrm{e}}=0.1$ [parts (a) and (c)] and $F_{\mathrm{e}}=0.3$ [parts (b) and (d)]. Top panels: plots of the local nematic order parameter $S(x, z)$ (see attached bar) and the nematic director $\widehat{\boldsymbol{n}}_{+}$(dashes). The arrows indicate the direction of the flow. Bottom panels: Parts (c) and (d) show plots of the local biaxiality order parameter $\xi(x, z)$ (see attached bar) and of the local eigenvector $\widehat{\boldsymbol{n}}_{-}$(dashes). In parts (c) and (d) the strength of the body force is the same as in the corresponding parts (a) and (b) of the figure. Plots in parts (a)-(d) have been obtained for $\varepsilon_{\text {opt }}=5.0$ and $T_{\text {in }}=1.0$ and $T_{\text {out }}=0.9$.

mesogens. Generally speaking, if a stationary nonequilibrium flow has been initiated, the symmetry is broken along the flow direction such that $S, \xi, \widehat{\boldsymbol{n}}_{+}$, and $\widehat{\boldsymbol{n}}_{-}$may now differ between the upstream and downstream side of the laser beam.

As before in thermodynamic equilibrium, the nematic halo visible in the plot of $S$ demarcates the region in which $S$ decreases on account of the optical torque. As in Figure 4(a) the decrease of $S$ indicates the region of reorientation of the mesogens and their beginning alignment with the $y$-direction. However, in the presence of flow the halo appears to be deformed towards the downstream side. This deformation can already be detected in Figure 8(a) and is clearly visible in Figure 8(b).

The corresponding maps of $\xi$ and $\widehat{\boldsymbol{n}}_{-}$in Figure 8(c,d) show that the biaxiality on the upstream side is lowered. This effect is already seen in Figure 8(c) but can easily be detected in Figure $8(\mathrm{~d})$; $\xi$ is rather low on the upstream compared with the downstream side. This phenomenon can be rationalised as follows.

The plot of $\widehat{\boldsymbol{n}}_{-}$in Figure 8(c) shows that this eigenvector field is slightly bent towards the $z$-axis on the upstream side as the solid walls are approached. This implies that $\widehat{\boldsymbol{n}}_{-}$does not exactly coincide with $\widehat{\boldsymbol{e}}_{\mathrm{x}}$ and therefore a second symmetry axis is less well defined.

As $\Delta x \rightarrow 0^{-}$the mesogens are increasingly aligned with the $y$-axis. As explained before, this is inferred from the absence of the small dashes representing $\widehat{\boldsymbol{n}}_{+}$in Figure 8(b). The corresponding plot in Figure 8(d) indicates that by the time the mesogens are moving out of the laser beam the nematic director $\widehat{\boldsymbol{n}}_{+}$is aligned nearly perfectly with the $z$-axis and so is $\widehat{\boldsymbol{n}}_{-}$with the $x$-axis. Thus, the direction of $\widehat{\boldsymbol{n}}_{-}$becomes again the axis of rotation so that together with $\widehat{\boldsymbol{n}}_{+}$we have a system with biaxial symmetry. This is the reason why $\xi$ is relatively large on the downstream side of the halo visible in Figure 8(d).

A somewhat peculiar feature visible in Figure 8(d) are two bands of low to moderate biaxiality $\xi \simeq 0.08$ running across the entire system along the $\Delta x$-axis. These bands can solely be ascribed to the presence of the flow as the topological similarity between the plots in Figures 8(d) and 9 suggests. The biaxial bands can be rationalised by the plots of components of $\widehat{\boldsymbol{n}}_{+}$and $\widehat{\boldsymbol{n}}_{-}$presented in Figure 10(a,b).

The plot in Figure 10(a) shows that $n_{z,+}$ is nearly one but exhibits two minima that are shifted symmetrically with respect to $z=0$. Because $n_{\mathrm{z},+} \approx 1.0$ the mesogens are nearly perfectly aligned with the $z$-axis. Moreover, if $n_{\mathrm{z},+}<1.0, n_{\mathrm{x},+}$ turns out to be nonzero and changes sign at $z=0\left(n_{\mathrm{y},+} \simeq 0.0\right.$ because $\widehat{\boldsymbol{n}}_{+}$is a unit vector). Because $n_{\mathrm{x},+} \ll n_{\mathrm{z},+}$ and because $n_{\mathrm{x},+}$ changes sign one readily concludes that the nematic director must be very slightly tilted towards the flow $(x-)$ direction.

It is instructive to contrast these observations with the spatial variation of components $n_{\mathrm{x},-}$ and $n_{\mathrm{z},-}$ shown in Figure 10(b). The relative magnitude of these two components of $\widehat{\boldsymbol{n}}_{-}$is inverted compared with the corresponding components of $\widehat{\boldsymbol{n}}_{+}$. The plots in Figure 10(b) show that $n_{z,-}$ is always positive but nearly vanishes. As before, $n_{z,-}$ is symmetric with respect to $z=0$ where it is zero. The other component $n_{\mathrm{x},-}$ is the one dominating $\widehat{\boldsymbol{n}}_{-}$and is also symmetric with respect to $z=0$ where it changes sign. These features indicate that $\widehat{\boldsymbol{n}}_{-}$ is largely aligned with the flow direction but also has a nonvanishing component $n_{\mathrm{y},-}$.

Thus, the substrate-induced alignment of $\widehat{\boldsymbol{n}}_{+}$with the $z$-axis on the one hand and the flow-induced alignment of $\widehat{\boldsymbol{n}}_{-}$with the $x$-axis on the other hand causes the observed 


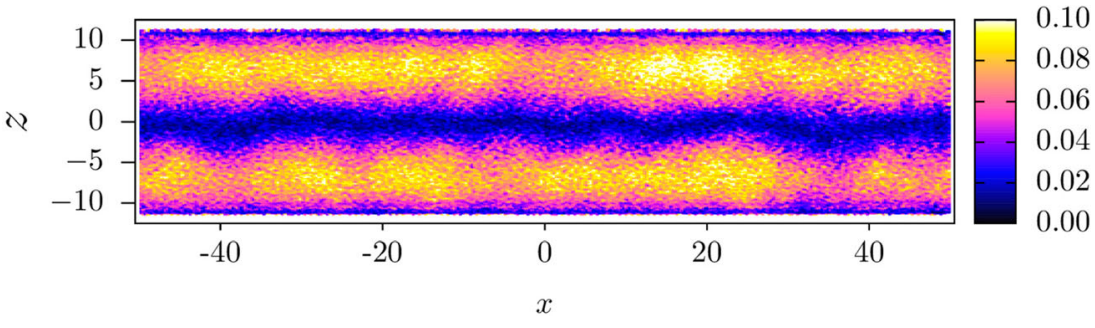

Figure 9. As Figure 8(d), but without the laser [i.e. for $T_{\text {in }}=T_{\text {out }}=0.9$ and $\varepsilon_{\text {opt }}=0.0$.]

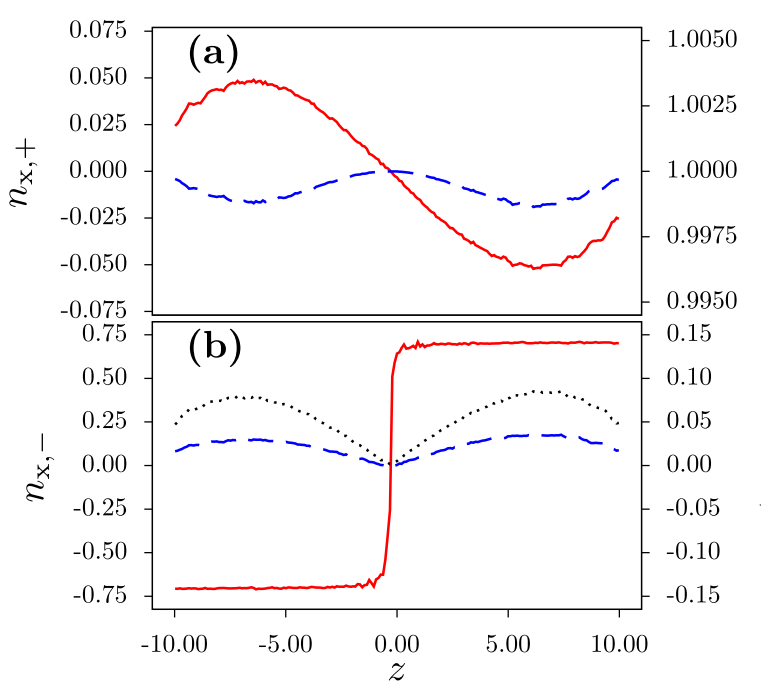

Figure 10. (a) Plots of $n_{x,+}(z)$ (_ ) (left ordinate) and $n_{z,+}(z)$ (--- ) (right ordinate) as function of the position $z$ between the solid walls. (b) as (a), but for $n_{x_{1}-}(z)$ and $n_{z,+}(z)$. In addition, the biaxiality order parameter $\xi(z)(.$.$) is also shown (right ordinate).$

biaxiality bands visible in Figures 8(d) and 9. As the plot of $\xi$ in Figure 10(b) reveals the biaxial order is fairly small. However, the bands visible in Figures 8(d) and 9 manifest themselves as the two maxima of $\xi$ in Figure 10(b).

If one now increases the strength of the optical torque by increasing $\varepsilon_{\text {opt }}$ in Equation (13) a couple of interesting features arise. First, comparing the plots in Figure 8(a,b)

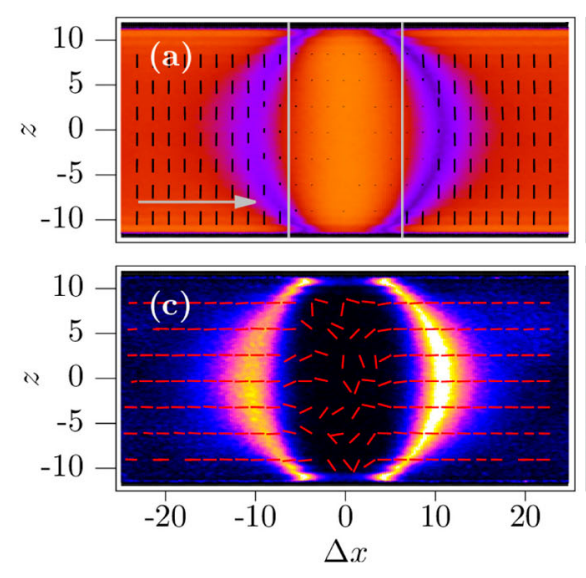

with their counterparts shown in Figure 11(a,b) one realises that the nematic order is enhanced inside the laser beam as far as the latter set of plots is concerned.

This is an immediate consequence of the larger value of $\varepsilon_{\text {opt }}$. The plots in Figure 3(b) for $\varepsilon_{\text {opt }}=5.0$ and $\varepsilon_{\text {opt }}=$ 15.0 reveal that the polar order inside the laser beam increases with $\varepsilon_{\text {opt }}$ as expected. Thus, the enhancement of $S$ in Figure 11 $(\mathrm{a}, \mathrm{b})$ reflects a more ordered polar nematic inside the laser beam.

Another consequence of increasing $\varepsilon_{\mathrm{opt}}$ is that the width of the nematic halo is larger if $\varepsilon_{\text {opt }}$ increases which can be seen by comparing the plot in Figures 8(a) and 11(a). This is also easy to understand because for two values of $\varepsilon_{\text {opt }}$ the optical torque will assume the same value at a larger value of $|\Delta x|$ if $\varepsilon_{\text {opt }}$ increases. Therefore, the reorientation of the mesogens from the $x-z$ plane to an alignment with the $y$-axis occurs at significantly larger values of $|\Delta x|$ in Figure 11(a) as opposed to Figure 8(a).

Consequently, the biaxial halo is also bigger in Figure 11(c) compared with the data plotted in Figure 8(c). This seems to be caused by the higher order at higher $\varepsilon_{\text {opt }}$ as the plots in Figures 3(b), 8(a,c), and 11(a,c) clearly show. This phenomenon can be explained because it takes more time for the mesogens to reorient themselves when the polarisation inside the laser beam is larger. Consequently the region over which this happens is widened if $\varepsilon_{\text {opt }}$ increases.

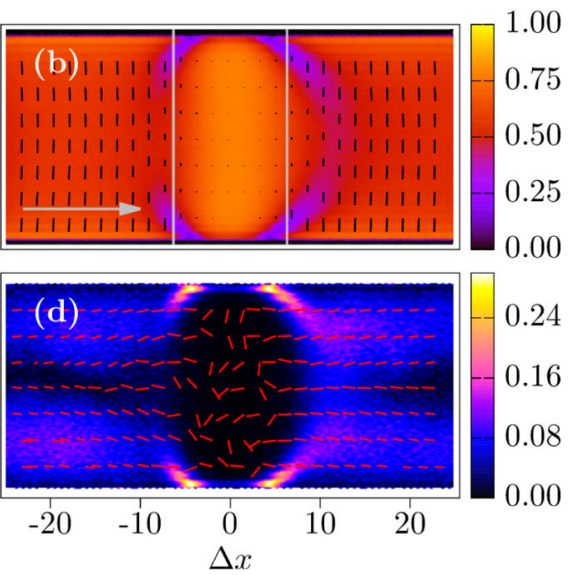

Figure 11. As Figure 8, but for $\varepsilon_{\mathrm{opt}}=15.0$ [see Equation (13)]. 
Last but not least, regions of biaxiality are absent in Figure 11(d) except for small regions in the immediate vicinity of the solid walls. If the flow is enhanced one realises that $\widehat{\boldsymbol{n}}_{-}$is different everywhere in the system. Hence, a common axis of rotation does not exist any longer and therefore there is no second symmetry axis. Consequently, the order is uniaxial almost everywhere as the plot in Figure 11(d) reveals.

\section{Discussion and conclusions}

Although the technological success of optofluidics has largely been realised through isotropic fluids, its full potential will depend on our ability to tailor and harnesses light-induced microscale flow properties of anisotropic fluids. With an eye for enabling future applications, in this work we investigate the properties of a simple model of a nematic liquid crystal in a steady-state nonequilibrium situation by means of NEMD simulations. To drive the system out of thermodynamic equilibrium a constant body force is applied to the mesogens located in a small volume at the beginning of the simulation cell. All results presented here pertain to the Stokes or creeping regime of laminar flow.

We then expose the liquid crystal to a laser field. Our laser is based upon a rather minimalistic model which accounts only for the most basic features, namely, local heating of the illuminated region and an optical torque that serves to change the nonpolar nematic structure of the liquid crystal to a polar one.

In principle, the simulations that have been carried out in this work would give one access to the dielectric anisotropy [40] which is linked to the refractive indices as a measurable quantity. As the dielectric anisotropy is also related to the alignment tensor one might envision to compare the present simulations with parallel experiments. However, a detailed analysis of these relationships is beyond the scope of the present article. Having demonstrated that our setup is capable to give physically meaningful results, one could think of extending the present model to one in which cholesteric structures are stable [54].

As pointed out in the book by de Gennes and Prost [43] it is possible to unwind the cholesteric helix by sufficiently strong electric or magnetic fields. Preliminary results by us show that this could very well be possible using optothermal conditions as well. Work along those lines is currently under way.

One could argue though that the dimensions of the constituents of our model laser are too tiny to represent a typical laser experiment. This would probably be the case if we take our mesogens to represent individual molecules in which our unit of length $\sigma$ would be of the order of several tenth of a nanometre. However, because of the small aspect ratio exceeding a value of 1.0 only slightly [35] it seems permissible to adopt the notion that our mesogens should, in fact, be perceived as nanoscopic droplets consisting of several individual molecules. The typical size of such a droplet could easily increase to several $10 \mathrm{~nm}$. This perception has been introduced originally by Maier and Saupe [55] and was adopted later by Steuer et al. [56] as well as by Schlotthauer et al. [57]. Based upon this supramolecular picture $[58,59]$ the width of our laser beam $\delta x$ would, in fact, correspond to a couple of hundred nanometres.

Similarly, we allow the temperature $T$ of the liquid crystal to increase on account of the laser by a small amount $\Delta T=0.1$ in dimensionless units. Based upon the assumption that our unit of energy $\varepsilon / k_{\mathrm{B}}=\mathcal{O}\left(10^{2}\right) \mathrm{K}$, $\Delta T$ would correspond to an increase of the temperature inside the laser beam by some ten $\mathrm{K}$.

The liquid crystal is placed between two plane parallel, atomically corrugated solid walls that we need for the mesogens to experience friction under nonequilibrium steady-state flow conditions. This friction is a necessary prerequisite to establish the typical parabolic velocity profiles characteristic of Poiseuille flow.

Outside of the illuminated area the mesogens are aligned with the $z$-axis and therefore exhibit homeotropic alignment with respect to the planes of the solid walls. Sufficiently far away from the laser both $\widehat{\boldsymbol{n}}_{+}$and $S$ are homogeneous and do not show any dependence on the specific location. As one approaches the laser the optical torque causes the mesogens to rotate out of the $x-z$ plane and to align with the $y$-axis. When this rotation sets in $S$ decreases because during this rotation the mesogens do not behave as a single entity. In other words, the rotation of the mesogens is not a coordinated process and the biaxiality order parameter $\xi$ increases simultaneously. However, this is apparently not true if $\boldsymbol{\tau}_{\text {opt }}$ is sufficiently large.

The appearance of a significant biaxiality can be explained as follows. When the mesogens begin to rotate out of the $x-z$ plane, it turns out that the axis of rotation is given by $\widehat{\boldsymbol{n}}_{-}$. Thus, the system has two principal symmetry axes, namely $\widehat{\boldsymbol{n}}_{+}$and $\widehat{\boldsymbol{n}}_{-}=\widehat{\boldsymbol{e}}_{\mathrm{x}}$ and therefore exhibits biaxial symmetry.

As soon as the alignment of the mesogens is complete the symmetry of the system inside the laser region becomes uniaxial again and the only constraint between the nematic director and the eigenvector associated with the smallest eigenvalue of $\mathbf{Q}$ is that $\widehat{\boldsymbol{n}}_{+} \cdot \widehat{\boldsymbol{n}}_{-}=0$. Hence, in the $x-z$ plane the distribution of the directions of $\widehat{\boldsymbol{n}}_{-}$ is isotropic.

Under relatively weak flow and for a weak optical torque this general picture prevails except that now the 
region of biaxiality turns out to be an approximately elliptic halo. This halo surrounds a region in which the directions of $\widehat{\boldsymbol{n}}_{-}$exhibit an isotropic arrangement across the $x-z$ plane. If the flow becomes stronger the halo becomes asymmetric. Biaxiality is reduced on the upstream and enhanced on the downstream side. The reduction on the upstream side is a consequence of a slight deviation between $\widehat{\boldsymbol{n}}_{-}$and the axis of rotation $\widehat{\boldsymbol{e}}_{\mathrm{x}}$.

The enhancement of biaxiality on the downstream side can also be rationalised fairly easily. First one realises that the optical torque aligns the mesogens nearly perfectly with the $y$-axis at the centre of the laser beam. Because $\widehat{\boldsymbol{n}}_{+} \cdot \widehat{\boldsymbol{n}}_{-}=0$, the eigenvector $\widehat{\boldsymbol{n}}_{-}$becomes aligned with the axis of rotation $\widehat{\boldsymbol{e}}_{\mathrm{x}}$. As the mesogens are being carried out of the laser beam by the flow and as they begin to rotate back to their original homeotropic alignment with the plane of the solid walls, the system again exhibits two well-defined principal symmetry axes. As a consequence the biaxial halo is more pronounced on the downstream side.

The situation becomes more complex if the strength of the optical torque increases [i.e. for larger values of $\varepsilon_{\text {opt }}$ in Equation (13)]. As we show in Section 5.3 increasing the optical torque has two main consequences. First, the (polar) nematic order inside the laser beam increases; second, the biaxial halo is shifted and broadened compared with the situation for a weaker optical torque. Thus, for a rather weak flow it takes the mesogens longer to assume a polar nematic order at the centre of the laser beam.

For stronger flow and for a stronger optical torque it turns out that the liquid crystal is uniaxially ordered almost everywhere in the system. This is because it turns out that a common axis of rotation does not longer exist. Therefore, a second symmetry axis, the necessary prerequisite for biaxiality, cannot be established.

We would also like to stress that for the present class of systems liquid-crystalline properties arise on account of strongly anisotropic attraction between mesogens that have a nearly spherical core. Both features make them quite distinct from more conventional model systems such as spherocylinders or the widely used Gay-Berne particles. In these latter models ordered mesophases arise mostly because of the shape anisometry of the mesogens. In our opinion it is fair to say that with the advent of the present class of models has significantly advanced the numerical study of liquid-crystalline materials and its various features.

The reason for this is twofold. First, on account of the nearly spherical core of the mesogens the systems are fairly easy to equilibrate and therefore they are computationally cheap. This is particularly important in the context of the present work where large systems had to be utilised. These large samples are required to have a sufficiently wide laser beam surrounded by sufficiently large bulk-like regions.

In addition, we are restricted to the small-flow regime to avoid spurious effects from the periodic boundary conditions and to make sure the velocity profiles generated are characteristic of Poiseuille flow. For slow flows the temporal evolution of the system is also slow and therefore the simulations have to be run for a long time to establish a stationary nonequilibrium situation and to sample quantities of interest with sufficiently good statistics. This is the second reason why one wishes to have a computationally cheap model system at hand.

At the same time it needs to be emphasised that despite their simplicity and computational convenience, the present class of model liquid crystals has repeatedly been shown to be sufficiently realistic. Examples include structures and the dynamics in nematic colloidal suspensions [52]. These structures were observed experimentally $[60,61]$ but remained unexplained for nearly twenty years. Another example concerns the development of defect topologies that form when a colloidal particle is immersed into a nematic or smectic $A$ carrier fluid [62]. Our theoretical results [63] are in good qualitative agreement with experimental findings $[64,65]$. Our last example concerns biaxial binary mixtures of two uniaxially symmetric compounds that pertain also to the present class of model systems [25].

\section{Disclosure statement}

No potential conflict of interest was reported by the authors.

\section{Funding}

AS was supported by the ATTRACT Investigator Grant of the Luxembourg National Research Fund [grant number A17/MS/11572821/MBRACE].

\section{ORCID}

Anupam Sengupta (D) http://orcid.org/0000-0001-5592-7864 Marco G. Mazza (D) http://orcid.org/0000-0002-5625-9121

Martin Schoen (D) http://orcid.org/0000-0002-6268-1775

\section{References}

[1] M. Kleman and O.D. Lavrentovich, Soft Matter Physics: An Introduction (Springer-Verlag, New York, 2003).

[2] P. Oswald and P. Pieranski, Nematic and Cholesteric Liquid Crystals: Concepts and Physical Properties Illustrated by Experiments (CRC Press, Boca Raton, 2005).

[3] Y.K. Kim, B. Senyuk and O.D. Lavrentovich, Nat. Commun. 3, 1133 (2012).

[4] E.K. Fleischmann, H.L. Liang, N. Kapernaum, F. Giesselmann, J. Lagerwall and R. Zentel, Nat. Commun. 3, 1178 (2012). 
[5] A. Sengupta, U. Tkalec, M. Ravnik, J.M. Yeomans, C. Bahr and S. Herminghaus, Phys. Rev. Lett. 110, 048303 (2013).

[6] A. Sengupta, S. Herminghaus and C. Bahr, Liq. Cryst. Rev. 2, 73-110 (2014).

[7] T. Stieger, H. Agha, M. Schoen, M.G. Mazza and A. Sengupta, Nat. Commun. 8, 15550 (2017).

[8] D. Psaltis, S.R. Quake and C. Yang, Nature 442 (7101), 381-386 (2006).

[9] J.G. Cuennet, A.E. Vasdekis, L. De Sio and D. Psaltis, Nat. Photonics 5, 234 (2011).

[10] A. Sengupta, S. Herminghaus and C. Bahr, Appl. Phys. Lett. 101, 164101 (2012).

[11] J.G. Cuennet, A.E. Vasdekis and D. Psaltis, Lab Chip 13, 2721-2726 (2013).

[12] M.J. Freiser, Phys. Rev. Lett. 24, 1041-1043 (1970).

[13] G.R. Luckhurst, Thin Solid Films 393, 40-52 (2001).

[14] B.R. Acharya, A. Primak and S. Kumar, Phys. Rev. Lett. 92, 145506 (2004).

[15] L.A. Madsen, T.J. Dingemans, M. Nakata and E.T. Samulski, Phys. Rev. Lett. 92, 145505 (2004).

[16] V. Prasad, S.W. Kang, K. Suresh, L. Joshi, Q. Wang and S. Kumar, J. Am. Chem. Soc. 127, 17224-17227 (2005).

[17] H. Mundoor, S. Park, B. Senyuk, H.H. Wensink and I.I. Smalyukh, Science 360, 768-771 (2018).

[18] R. Alben, J. Chem. Phys. 59, 4299-4304 (1973).

[19] A. Stroobants and H. Lekkerkerker, J. Phys. Chem. 88, 3669-3674 (1984).

[20] P. Palffy-Muhoray, J.R. de Bruyn and D.A. Dunmur, J. Chem. Phys. 82, 5294-5295 (1985).

[21] M.P. Allen, Liq. Cryst. 8, 499-511 (1990).

[22] A.G. Vanakaras and D.J. Photinos, Mol. Cryst. Liq. Cryst. 299, 65-71 (1997)

[23] L. Longa, J. Stelzer and D. Dunmur, J. Chem. Phys. 109, 1555-1566 (1998).

[24] R. Berardi and C. Zannoni, J. Chem. Phys. 113, 5971-5979 (2000).

[25] R.A. Skutnik, L. Lehmann, S. Püschel-Schlotthauer, G. Jackson, and M. Schoen, Mol. Phys. (2019). doi:10.1080/ 00268976.2019 .1581292 .

[26] J.J. Hunt, R.W. Date, B.A. Timimi, G.R. Luckhurst and D.W. Bruce, J. Am. Chem. Soc. 123, 10115-10116 (2001).

[27] A.G. Vanakaras, M.A. Bates and D.J. Photinos, Phys. Chem. Chem. Phys. 5, 3700-3706 (2003).

[28] R. Bartolino, T. Chiaranza, M. Meuti and R. Compagnoni, Phys. Rev. A 26, 1116-1119 (1982).

[29] A. Sengupta, Liq. Cryst. 41, 290-301 (2014).

[30] A. Sengupta, Int. J. Mol. Sci. 14, 22826-22844 (2013).

[31] L. Giomi, Ž. Kos, M. Ravnik and A. Sengupta, Proc. Natl. Acad. Sci. U.S.A. 114, E5771-E5777 (2017)

[32] C.G. Gray and K.E. Gubbins, Theory of Molecular Fluids, vol. 1 (Clarendon Press, Oxford, 1984).

[33] S. Hess and B. Su, Z. Naturforsch. A 54, 559-569 (1999).

[34] A.J. Stone, Mol. Phys. 36, 241-256 (1978).

[35] M. Greschek, M. Melle and M. Schoen, Soft Matter 6, 1898-1909 (2010).

[36] T. Stieger, Ph. D. thesis, Technische Universität Berlin, 2016.

[37] S.D. Stoyanov and R.D. Groot, J. Chem. Phys. 122, 114112 (2005).

[38] R.D. Groot, J. Chem. Theory Comput. 2, 568-574 (2006).
[39] T. Stieger, M. Schoen and M.G. Mazza, J. Chem. Phys. 140 054905 (2014).

[40] F. Simoni and O. Francescangeli, J. Phys.: Condens. Matter 11, R439-R486 (1999).

[41] H.J. Weber and G.B. Arfken, Essential Mathematical Methods for Physicists (Academic Press, San Diego, 2003).

[42] W.H. Press, S.A. Teukolsky, W.T. Vetterling and B.P. Flannery, Numerical Recipes: The Art of Scientific Computing, 3rd ed. (Cambridge University Press, Cambridge, 2007).

[43] P.G. de Gennes and J. Prost, The Physics of Liquid Crystals, 2nd ed. (Oxford Science Publications, Oxford, 1995).

[44] M. Gramzow and S.H.L. Klapp, Phys. Rev. E 75, 011605 (2007).

[45] S.M. Wandrei, R. Roth and M. Schoen, J. Chem. Phys. 149, 054704 (2018).

[46] G.K. Batchelor, An Introduction to Fluid Dynamics (Cambridge University Press, Cambridge, 2002).

[47] D.A. McQuarrie, Statistical Mechanics (Harper \& Row, New York, 1976).

[48] B.D. Todd, D.J. Evans and P.J. Daivis, Phys. Rev. E 52, 1627-1638 (1995).

[49] K.P. Travis and K.E. Gubbins, J. Chem. Phys. 112, 1984-1994 (2000).

[50] M.P. Allen and D.J. Tildesley, Computer Simulation of Liquids, 2nd ed. (Oxford University Press, Oxford, 2017).

[51] J. Ilnytskyi and M.R. Wilson, Comput. Phys. Commun. 134, 23-32 (2001).

[52] S. Püschel-Schlotthauer, T. Stieger, M. Melle, M.G. Mazza and M. Schoen, Soft Matter 12, 469-480 (2016).

[53] L.D. Landau and E.M. Lifshitz, Course of Theoretical Physics. Fluid Mechanics, 3rd ed., vol. 6 (Pergamon Press, Oxford, 1966).

[54] M. Melle, M. Theile, C.K. Hall and M. Schoen, Int. J. Mol. Sci. 14, 17584-17607 (2013).

[55] W. Maier and A. Saupe, Z. Naturforsch. A 15, 287-292 (1960).

[56] H. Steuer, S. Hess and M. Schoen, Physica A 328, 322-334 (2003).

[57] S. Schlotthauer, R.A. Skutnik, T. Stieger and M. Schoen, J. Chem. Phys. 142, 194704 (2015).

[58] J.M. Lehn, Angew. Chem. 102, 1347-1362 (1990).

[59] F. Vögtle, Supramolekulare Chemie: Eine Einführung (Springer-Verlag, Berlin, 2013).

[60] P. Poulin and D.A. Weitz, Phys. Rev. E 57, 626 (1998).

[61] I.I. Smalyukh, O.D. Lavrentovich, A.N. Kuzmin, A.V. Kachynski and P.P.N. Prasad, Phys. Rev. Lett. 95, 157801 (2005).

[62] S. Püschel-Schlotthauer, Ph. D. thesis, Technische Universität Berlin, 2017.

[63] S. Püschel-Schlotthauer, V.M. Turrión, C.K. Hall, M.G. Mazza and M. Schoen, Langmuir 33, 2222-2234 (2017).

[64] K.P. Zuhail, P. Sathyanarayana, D. Seč, S. Čopar, M. Škarabot, I. Muševič and S.S. Dhara, Phys. Rev. E 91, 030501 (2015).

[65] K.P. Zuhail and S. Dhara, Appl. Phys. Lett. 106, 211901 (2015).

[66] J.P. Hansen and I.R. McDonald, Theory of Simple Liquids, 3rd ed. (Elsevier, London, 2006).

[67] R. Eppenga and D. Frenkel, Mol. Phys. 52, 1303-1334 (1984). 


\section{Appendices}

\section{Appendix 1. Treatment of the orientation dependence of interactions}

In this Appendix we give an account of the derivation of $u$ and $\Psi$ in Equations (6) and (7), respectively. Because the set of basis functions $\left\{\mathcal{Y}_{T \widetilde{m}}\right\}$ is complete, the expansion of $u_{\text {aniso }}$ in Equation (4) is exact. However, Equation (4) is not very useful on account of the fact that the number of terms involved in the sixfold summation over $l_{1}, l_{2}, l, m_{1}, m_{2}$, and $m$ grows very quickly. Thus, Equation (4) becomes prohibitively cumbersome even if one truncates it at relatively small values of $l_{1}$, $l_{2}$, and $l$. Therefore, additional, physically sensible assumptions need to be introduced to render Equation (4) computationally tractable.

We are seeking an orientation dependence of $u_{\text {aniso }}$ that is characterised by two features, namely

(1) The resulting potential should involve a contribution that depends on the relative orientations $\omega_{i}$ and $\omega_{j}$ but should not depend on the orientations of both mesogens with respect to the orientation of the distance vector $\widehat{\boldsymbol{r}}_{i j}$ connecting their centres of mass.

(2) There should be also a contribution involving the orientation of either mesogen $i$ or $j$ with respect to the orientation of $\widehat{\boldsymbol{r}}_{i j}$.

Because of these two requirements we can split $u_{\text {aniso }}$ into two contributions according to

$$
u_{\text {aniso }}\left(\boldsymbol{r}_{i j}, \omega_{i}, \omega_{j}\right)=u^{(1)}\left(r_{i j}, \omega_{1}, \omega_{2}\right)+u^{(2)}\left(\boldsymbol{r}_{i j}, \omega_{i}, \omega_{j}\right),
$$

where the superscripts refer to assumption (1) and (2), respectively. Because of this decomposition and because of Equation (4) we now have to consider two separate expansions of $u^{(1)}$ and $u^{(2)}$ in the basis of the rotational invariants.

On account of condition (1), $u^{(1)}$ can depend only on $r_{i j}$ at the most. This implies that for any fixed orientation of mesogens $i$ and $j, u^{(1)}$ is effectively isotropic. In other words, $\Phi_{l_{1} l_{2} l}$ cannot depend on $\omega_{i j}$ which suggests that $l=m=0$ in Equation (5). Under this premise nonzero Clebsch-Gordan coefficients are obtained only if the two selection rules

- $m_{1}+m_{2}=m$ [see Equation (A.130) of Ref. [32]] and

- $\left|l_{1}-l_{2}\right| \leq l \leq l_{1}+l_{2}$ [triangle inequality, see Equation (A.131) of Ref. [32]]

are satisfied simultaneously. If $l=m=0$ the first of these reduces to $m_{1}=-m_{2}$. In addition, for $l=0$ the triangle inequality can only be satisfied if $l_{1}=l_{2}$. Using then Equation (A.157) of Ref. [32] we obtain from Equations (4) and (5)

$$
\begin{aligned}
& \frac{1}{\sqrt{4 \pi}} \sum_{l=0}^{\infty} \frac{u_{l l 0}\left(r_{i j}\right)}{\sqrt{2 l+1}} \sum_{m=-l}^{l}(-1)^{l+m} \mathcal{Y}_{l m}\left(\omega_{i}\right) \mathcal{Y}_{l \underline{m}}\left(\omega_{j}\right) \\
& =\frac{1}{\sqrt{4 \pi}} \sum_{l>0}^{\infty} \frac{u_{l l 0}\left(r_{i j}\right)}{\sqrt{2 l+1}} \sum_{m=-l}^{l}(-1)^{l+m} \mathcal{Y}_{l m}\left(\omega_{i}\right) \mathcal{Y}_{l \underline{m}}\left(\omega_{j}\right) \\
& \quad+\frac{1}{(4 \pi)^{3 / 2}} u_{000}\left(r_{i j}\right)
\end{aligned}
$$

where $\underline{m}=-m$ and $\mathcal{Y}_{00}=1 / \sqrt{4 \pi}$ has also been used. Notice, that on the second and third lines of Equation (A2) the isotropic contribution $u_{000}$ is treated separately.

Invoking now the relation $(-1)^{m} \mathcal{Y}_{l \underline{m}}=\mathcal{Y}_{l m}^{*}$ together with the addition theorem for spherical harmonics [see Equation (A.33) of Ref. [32]] allows us to rewrite the previous expression for $u^{(1)}$ more compactly as

$$
\begin{aligned}
& \sum_{l>0}^{\infty} \frac{(-1)^{l} \sqrt{2 l+1}}{(4 \pi)^{3 / 2}} u_{l l 0}\left(r_{i j}\right) P_{l}(x)+\frac{1}{(4 \pi)^{3 / 2}} u_{000}\left(r_{i j}\right) \\
& \quad=u^{(1)}\left(r_{i j}, \omega_{i}, \omega_{j}\right)+\frac{1}{(4 \pi)^{3 / 2}} u_{000}\left(r_{i j}\right)
\end{aligned}
$$

where $P_{l}$ is a Legendre polynomial of order $l$ and $x=\widehat{\boldsymbol{u}}_{i} \cdot \widehat{\boldsymbol{u}}_{j}$ is the cosine of the angle between $\widehat{\boldsymbol{u}}_{i}$ and $\widehat{\boldsymbol{u}}_{j}$ [see Equation (2)].

In addition, we require $u^{(1)}$ to be invariant if mesogen $i$ (or $j$ ) is inverted such that $\gamma_{i j} \rightarrow \gamma_{i j}^{\prime}=\gamma_{i j}+\pi$. This invariance of $u^{(1)}$ reflects the head-tail symmetry typical of many mesogens[43]. Using the well-known addition theorems for trigonometric functions it is easy to verify that this transformation yields $x^{\prime}=\cos \gamma_{i j}^{\prime}=-x$.

For odd integers $l, P_{l}$ contains only odd powers of $x$ whereas it consists only of even powers of $x$ (and a constant) if $l$ is even [see Equations (A.8) and (A.9) of Ref. [32]]. Thus, to preserve the desired invariance of $u^{(1)}$, only terms for even $l$ should be retained in the sum on the righthand side of Equation (A3); terms for odd $l$ are being discarded henceforth.

Turning now to condition (2) one first realises that in the analogous expansion of $u^{(2)}, l$ must not be zero. If it were, we would again have $l=m=0$. Consequently, $u^{(2)}$ would be independent of $\boldsymbol{r}_{i j}$ which is inconsistent with condition (2). We also impose a symmetry constraint on $u^{(2)}$. Because the mesogens are achiral, $u^{(2)}$ should be invariant under the transformation $\omega_{i j} \rightarrow \omega_{i j}^{\prime}=-\omega_{i j}$. In other words, if mesogens $i$ and $j$ interchange their centre-of-mass position such that $\boldsymbol{r}_{i j} \rightarrow$ $\boldsymbol{r}_{i j}^{\prime}=-\boldsymbol{r}_{i j}, u^{(2)}$ should remain unaffected. The inversion parity of spherical harmonics [see Equation (A47) of Ref. [32]] then suggests that $l$ must be even and therefore must satisfy the inequality $l \geq 2$.

Next, we remind ourselves that from condition (2) the orientation dependence of $u^{(2)}$ should be such that it can be decomposed into separate contributions where either the orientation of mesogen $i$ with respect to $\widehat{\boldsymbol{r}}_{i j}$ or that of mesogen $j$ matters. This prompts us to write the expansion of $u^{(2)}$ more explicitly as

$$
\begin{aligned}
u^{(2)}\left(\boldsymbol{r}_{i j}, \omega_{1}, \omega_{2}\right)= & \sum_{\substack{l_{1}, l \\
l \geq 2}} u_{l_{1} 0 l}\left(r_{i j}\right) \Phi_{l_{1} 0 l}\left(\omega_{i}, \omega_{j}, \omega_{i j}\right) \\
& +\sum_{\substack{l_{2}, l \\
l \geq 2}} u_{0 l_{2} l}\left(r_{i j}\right) \Phi_{0 l_{2} l}\left(\omega_{i}, \omega_{j}, \omega_{i j}\right) .
\end{aligned}
$$

The triangle inequality therefore suggests that $l_{1}=l$ in the first term on the righthand side of this expression. By the same token, $l_{2}=l$ must hold for the second term. Thus, both double sums collapse to single ones. 
We now invoke the definition of rotational invariants given in Equation (5) which gives

$$
\begin{aligned}
\Phi_{l 0 l}\left(\omega_{i}, \omega_{j}, \omega_{i j}\right) & =\frac{1}{\sqrt{4 \pi}} \sum_{m} C(l 0 l ; m 0 m) \mathcal{Y}_{l m}\left(\omega_{i}\right) \mathcal{Y}_{l m}^{*}\left(\omega_{i j}\right) \\
& =\frac{1}{\sqrt{4 \pi}} \sum_{m} \mathcal{Y}_{l m}\left(\omega_{i}\right) \mathcal{Y}_{l m}^{*}\left(\omega_{i j}\right) \\
& =\frac{2 l+1}{(4 \pi)^{3 / 2}} P_{l}\left(x_{i}\right),
\end{aligned}
$$

where $\mathcal{Y}_{00}=1 / \sqrt{4 \pi}, C(l 0 l ; m 0 m)=1$, and the addition theorem for spherical harmonics have also been invoked [see Equations (A.33), (A.62), and (A.156) of Ref. [32]]. In Equation (A5), $x_{i}=\widehat{\boldsymbol{u}}_{i} \cdot \widehat{\boldsymbol{r}}_{i j}$ denotes the cosine of the angle between the unit vector $\widehat{\boldsymbol{u}}_{i}$ and $\widehat{\boldsymbol{r}}_{i j}$. A similar expression is obtained for $\Phi_{0 l l}$ where, however $x_{i}$ is replaced by $x_{j}=\widehat{\boldsymbol{u}}_{j}$. $\widehat{\boldsymbol{r}}_{i j}$ and $C(0 \mathrm{ll} ; 0 \mathrm{~mm})=C(\mathrm{l0l} ; \mathrm{m0m})$ [see Equation (A.134) of Ref. [32]] has been invoked. We can then rewrite Equation (A4) more compactly as

$$
\begin{aligned}
u^{(2)}\left(\boldsymbol{r}_{i j}, \omega_{1}, \omega_{2}\right) & \\
= & \sum_{\substack{l \geq 2 \\
l \text { even }}} \frac{2 l+1}{(4 \pi)^{3 / 2}}\left[u_{l 0 l}\left(r_{i j}\right) P_{l}\left(x_{i}\right)+u_{0 l l}\left(r_{i j}\right) P_{l}\left(x_{j}\right)\right] .
\end{aligned}
$$

It is clear from Equations (A2) and (A4) that we would still need to consider an infinite number of terms in principle. Because this is clearly impossible we limit our treatment to the leading term describing the orientation dependence of $u_{\text {aniso }}$. From Equations (A1), (A2), and (A6) we then have

$$
\begin{aligned}
u_{\text {aniso }}\left(\boldsymbol{r}_{i j}, \omega_{i}, \omega_{j}\right)= & \frac{1}{(4 \pi)^{3 / 2}}\left\{\sqrt{5} u_{220}\left(r_{i j}\right) P_{2}(x)\right. \\
& \left.+5\left[u_{202}\left(r_{i j}\right) P_{2}\left(x_{i}\right)+u_{022}\left(r_{i j}\right) P_{2}\left(x_{j}\right)\right]\right\} .
\end{aligned}
$$

So far nothing is known about the expansion coefficients $u_{000}, u_{220}, u_{202}$, and $u_{022}$. However, introducing as our last assumption that mesogens $i$ and $j$ interact via dispersion interactions only, all four expansion coefficients are proportional to $-1 / r_{i j}^{6}$ [see Equations (2.226), (2.228), and (2.230) of Ref. [32]]. The coefficient of proportionality involves the average ionisation energy of the interacting pair of mesogens times their principal axes static polarisability components [32] and thus has units of energy $\times(\text { length })^{6}$. From this dimensional argument and putting together all the remaining, purely numerical prefactors it seems quite reasonable to express the expansion coefficients as

$$
\begin{aligned}
& u_{000}\left(r_{i j}\right)=-4 \varepsilon\left(\frac{\sigma}{r_{i j}}\right)^{6}, \\
& u_{220}\left(r_{i j}\right)=-4 \varepsilon \varepsilon^{\prime}\left(\frac{\sigma}{r_{i j}}\right)^{6}, \\
& u_{202}\left(r_{i j}\right)=-4 \varepsilon \varepsilon^{\prime \prime}\left(\frac{\sigma}{r_{i j}}\right)^{6}, \\
& u_{022}\left(r_{i j}\right)=-4 \varepsilon \varepsilon^{\prime \prime}\left(\frac{\sigma}{r_{i j}}\right)^{6} .
\end{aligned}
$$

At this stage it seems sensible to combine the isotropic contribution given by the expression in Equation (A8a) with the soft-sphere potential introduced in Equation (3). This gives the well-known Lennard-Jones potential function.

The dimensionless constants $\varepsilon^{\prime}$ and $\varepsilon^{\prime \prime}$ appearing in Equations (A8b)-(A8d) take notice of the fact that different combinations of the principal axes static polarisability components arise in the expressions for the expansion coefficients [32]. Thus, from Equations (1), (A7), and (A8) we finally arrive at the expressions given in Equations (6) and (7).

\section{Appendix 2. Derivation of the local alignment tensor}

In this Appendix we seek to derive a molecular expression for the local alignment tensor. We begin by considering first the generic distribution function [see Equation (3.91) of Ref. [32]] defined as

$$
\rho\left(\boldsymbol{r}^{N}, \omega^{N}\right)=\frac{N !}{Z} \exp \left[-\beta \mathcal{U}\left(\boldsymbol{r}^{N}, \omega^{N}\right)\right],
$$

where

$$
Z=\iint \mathrm{d} \boldsymbol{r}^{N} \mathrm{~d} \omega^{N} \exp \left[-\beta \mathcal{U}\left(\boldsymbol{r}^{N}, \omega^{N}\right)\right],
$$

and $\boldsymbol{r}^{N}=\left\{\boldsymbol{r}_{1}, \boldsymbol{r}_{1}, \ldots, \boldsymbol{r}_{N}\right\}$ and $\omega^{N}=\left\{\omega_{1}, \omega_{1}, \ldots, \omega_{N}\right\}$ are shorthand notations for the set of $N$ centre-of-mass positions and orientations, respectively; similarly, $\mathrm{d} \boldsymbol{r}^{N} \mathrm{~d} \omega^{N}=\mathrm{d} \boldsymbol{r}_{1} \ldots \mathrm{d} \boldsymbol{r}_{N} \mathrm{~d} \omega_{1}$ $\ldots \mathrm{d} \omega_{N}$. In Equations (A9) and (A10), $\beta=1 / k_{\mathrm{B}} T\left(k_{\mathrm{B}}\right.$ is Boltzmann's constant and $T$ is the temperature), and

$$
\begin{aligned}
\mathcal{U}\left(\boldsymbol{r}^{N}, \omega^{N}\right)= & \sum_{i=1}^{N-1} \sum_{j=i+1}^{N} u\left(\boldsymbol{r}_{i j}, \omega_{i}, \omega_{j}\right) \\
& +\sum_{k=1}^{2} \sum_{i=1}^{N} \sum_{j=1}^{N^{(k)}} u_{\mathrm{fs}}\left(r_{i j}^{(k)}\right),
\end{aligned}
$$

where $N^{(k)}$ is the number of solid atoms in wall $k$ and $u$ and $u_{\mathrm{fs}}$ are given by Equations (6) and (15), respectively.

From Equation (A9) we obtain the $n$-particle generic distribution function by integrating over the subsets $r^{N-n}=$ $\left\{\boldsymbol{r}_{n+1}, \boldsymbol{r}_{n+2}, \ldots, \boldsymbol{r}_{N}\right\} \subseteq \boldsymbol{r}^{N}$ and $\omega^{N-n}=\left\{\omega_{n+1}, \omega_{n+2}, \ldots, \omega_{N}\right\}$ $\subseteq \omega^{N}$ according to

$$
\begin{aligned}
\rho\left(\boldsymbol{r}^{n}, \omega^{n}\right)= & \frac{N !}{(N-n) !} \frac{1}{Z} \iint \mathrm{d} \boldsymbol{r}^{N-n} \mathrm{~d} \omega^{N-n} \\
& \times \exp \left[-\beta \mathcal{U}\left(\boldsymbol{r}^{N}, \omega^{N}\right)\right],
\end{aligned}
$$

where the combinatorial factor $N ! /(N-n)$ ! is the number of ways in which subsets $\boldsymbol{r}^{n}$ and $\omega^{n}$ can be formed within the sets $\boldsymbol{r}^{N}$ and $\omega^{N}$, respectively [32].

Consider now

$$
\begin{aligned}
\langle\delta(\boldsymbol{r} & \left.\left.-\boldsymbol{r}_{1}\right) \delta\left(\omega-\omega_{1}\right)\right\rangle \\
= & \frac{1}{Z} \iint \mathrm{d} \boldsymbol{r}^{N} \mathrm{~d} \omega^{N} \delta\left(\boldsymbol{r}-\boldsymbol{r}_{1}\right) \delta\left(\omega-\omega_{1}\right) \\
& \times \exp \left[-\beta \mathcal{U}\left(\boldsymbol{r}^{N}, \omega^{N}\right)\right] \\
= & \frac{1}{Z} \iint \mathrm{d} \boldsymbol{r}^{N-1} \mathrm{~d} \omega^{N-1} \\
& \times \exp \left[-\beta \mathcal{U}\left(\boldsymbol{r}, \boldsymbol{r}^{N-1}, \omega, \omega^{N-1}\right)\right],
\end{aligned}
$$

where $\langle\ldots\rangle$ denotes the ensemble average of the product of the two Dirac $\delta$-functions. 
Following the arguments put forth in the book of Hansen and McDonald (see [66, Chapter 2.5]) one could replace $\boldsymbol{r}_{1}$ and $\omega_{1}$ in the arguments of the Dirac $\delta$-functions by any corresponding members of the sets $\boldsymbol{r}^{N}$ and $\omega^{N}$ without altering the ensemble average on the last line of Equation (A13). Thus, by summing up all these equivalent integrals one would have $N$ times the expression given in Equation (A13). In fact, with the aid of Equation (A12) $(n=1)$ it immediately follows that

$$
\rho(\boldsymbol{r}, \omega)=\sum_{i=1}^{N}\left\langle\delta\left(\boldsymbol{r}-\boldsymbol{r}_{i}\right) \delta\left(\omega-\omega_{i}\right)\right\rangle .
$$

To obtain a molecular expression for the local density we then integrate Equation (A13) according to

$$
\begin{aligned}
\rho(\boldsymbol{r})= & \int \mathrm{d} \omega \rho(\boldsymbol{r}, \omega) \\
= & \frac{N}{Z} \int \mathrm{d} \omega \iint \mathrm{d} \boldsymbol{r}^{N-1} \mathrm{~d} \omega^{N-1} \\
& \times \exp \left[-\beta \mathcal{U}\left(\boldsymbol{r}, \boldsymbol{r}^{N-1}, \omega, \omega^{N-1}\right)\right] \\
= & \frac{N}{Z} \iint \mathrm{d} \boldsymbol{r}^{N-1} \mathrm{~d} \omega^{N} \exp \left[-\beta \mathcal{U}\left(\boldsymbol{r}, \boldsymbol{r}^{N-1}, \omega^{N}\right)\right]
\end{aligned}
$$

which is equivalent to Equation (3.100) of Ref. [32]. Equation (A15) can be interpreted as the local density of the liquid crystal because it follows from Equation (A12) together with Equation (A15) that

$$
\int \mathrm{d} \boldsymbol{r} \rho(\boldsymbol{r})=N
$$

This is because for $n=1$ an additional integration over the one remaining centre-of-mass position the integral on the righthand side of Equation (A9) is equal to $Z$ which consequently cancels between numerator and denominator and therefore gives $N ! /(N-1) !=N$.

To proceed we introduce the single-mesogen alignment tensor defined as

$$
\mathbf{q}\left(\omega_{1}\right)=\frac{1}{2}\left[3 \widehat{\boldsymbol{u}}\left(\omega_{1}\right) \widehat{\boldsymbol{u}}\left(\omega_{1}\right)-\mathbf{1}\right],
$$

where $\mathbf{1}$ is the unit tensor. As its globally defined counterpart $\mathbf{Q}$ introduced quite some time ago by Eppenga and Frenkel [67], $\mathbf{q}$ is a second-rank, symmetric, and traceless tensor that can be represented by a $3 \times 3$ matrix.

We are now seeking to compute the ensemble average

$$
\begin{gathered}
\left\langle\mathbf{q}\left(\omega_{1}\right) \delta\left(\boldsymbol{r}-\boldsymbol{r}_{1}\right) \delta\left(\omega-\omega_{1}\right)\right\rangle \\
=\frac{1}{Z} \iint \mathrm{d} \boldsymbol{r}^{N} \mathrm{~d} \omega^{N} \mathbf{q}\left(\omega_{1}\right)
\end{gathered}
$$

$$
\begin{aligned}
& \times \delta\left(\boldsymbol{r}-\boldsymbol{r}_{1}\right) \delta\left(\omega-\omega_{1}\right) \exp \left[-\beta \mathcal{U}\left(\boldsymbol{r}^{N}, \omega^{N}\right)\right] \\
= & \frac{1}{Z} \iint \mathrm{d} \boldsymbol{r}^{N-1} \mathrm{~d} \omega^{N-1} \mathbf{q}(\omega) \\
& \times \exp \left[-\beta \mathcal{U}\left(\boldsymbol{r}, \boldsymbol{r}^{N-1}, \omega, \omega^{N-1}\right)\right] .
\end{aligned}
$$

By the same token as before one realises that any one of the members of the sets $r^{N}$ and $\omega^{N}$ could have been picked in evaluating the ensemble average in Equation (A18). Integrating Equation (A18) over the one remaining orientation as in Equation (A15) we compute

$$
\begin{aligned}
\frac{1}{\rho(\boldsymbol{r})} & \int \mathrm{d} \omega^{\prime} \sum_{i=1}^{N}\left\langle\mathbf{q}\left(\omega_{i}\right) \delta\left(\boldsymbol{r}-\boldsymbol{r}_{i}\right) \delta\left(\omega^{\prime}-\omega_{i}\right)\right\rangle \\
= & \frac{1}{\rho(\boldsymbol{r})} \frac{N}{Z} \int \mathrm{d} \omega^{\prime} \iint \mathrm{d} \boldsymbol{r}^{N-1} \mathrm{~d} \omega^{N-1} \mathbf{q}\left(\omega^{\prime}\right) \\
& \times \exp \left[-\beta \mathcal{U}\left(\boldsymbol{r}, \boldsymbol{r}^{N-1}, \omega^{N}\right)\right],
\end{aligned}
$$

where we use the notation $\omega^{\prime}$ to indicate that the set $\omega^{N-1}$ does not contain the orientation of one of the mesogens which has been picked at will. Thus, the integrations over orientations are carried out for all the remaining $N-1$ mesogens but that of the chosen one. At this stage it is convenient to introduce the probability density

$$
P\left(\boldsymbol{r}, \omega^{\prime}\right)=\frac{\iint \mathrm{d} \boldsymbol{r}^{N-1} \mathrm{~d} \omega^{N-1} \exp \left[-\beta \mathcal{U}\left(\boldsymbol{r}, \boldsymbol{r}^{N-1}, \omega^{N}\right)\right]}{\iint \mathrm{d} \boldsymbol{r}^{N-1} \mathrm{~d} \omega^{N} \exp \left[-\beta \mathcal{U}\left(\boldsymbol{r}, \boldsymbol{r}^{N-1}, \omega^{N}\right)\right]}
$$

as a measure of finding the centre of mass of a mesogen in a small volume $\mathrm{d} \boldsymbol{r}$ centred on $\boldsymbol{r}$ with an orientation within an interval $\mathrm{d} \omega^{\prime}$ around $\omega^{\prime}$. With this quantity we can rewrite Equation (A19) as

$$
\begin{aligned}
\mathbf{Q}(\boldsymbol{r})= & \int \mathrm{d} \omega \mathbf{q}(\omega) P(\boldsymbol{r}, \omega) \\
= & \frac{1}{\rho(\boldsymbol{r})} \int \mathrm{d} \omega \sum_{i=1}^{N}\left\langle\mathbf{q}\left(\omega_{i}\right)\right. \\
& \left.\times \delta\left(\boldsymbol{r}-\boldsymbol{r}_{i}\right) \delta\left(\omega-\omega_{i}\right)\right\rangle,
\end{aligned}
$$

where $\mathbf{Q}$ is the local alignment tensor and we have changed the notation such that $\omega^{\prime} \rightarrow \omega$. During a simulation the far righthand side of Equation (A21) is computed as a histogram of instantaneous values of $\mathbf{q}$. 